\title{
Sialotranscriptomics of Rhipicephalus zambeziensis reveals intricate expression profiles of secretory proteins and suggests tight temporal transcriptional regulation during blood-feeding
}

Minique Hilda de Castro ${ }^{1,2,3}$, Daniel de Klerk ${ }^{1}$, Ronel Pienaar ${ }^{1,4}$, D Jasper G Rees ${ }^{2,3}$ and Ben J Mans 1,3,4* $^{1,4^{*}}$

\begin{abstract}
Background: Ticks secrete a diverse mixture of secretory proteins into the host to evade its immune response and facilitate blood-feeding, making secretory proteins attractive targets for the production of recombinant anti-tick vaccines. The largely neglected tick species, Rhipicephalus zambeziensis, is an efficient vector of Theileria parva in southern Africa but its available sequence information is limited. Next generation sequencing has advanced sequence availability for ticks in recent years and has assisted the characterisation of secretory proteins. This study focused on the de novo assembly and annotation of the salivary gland transcriptome of $R$. zambeziensis and the temporal expression of secretory protein transcripts in female and male ticks, before the onset of feeding and during early and late feeding.

Results: The sialotranscriptome of $R$. zambeziensis yielded 23,631 transcripts from which 13,584 non-redundant proteins were predicted. Eighty-six percent of these contained a predicted start and stop codon and were estimated to be putatively full-length proteins. A fifth (2569) of the predicted proteins were annotated as putative secretory proteins and explained $52 \%$ of the expression in the transcriptome. Expression analyses revealed that 2832 transcripts were differentially expressed among feeding time points and 1209 between the tick sexes. The expression analyses further indicated that $57 \%$ of the annotated secretory protein transcripts were differentially expressed. Dynamic expression profiles of secretory protein transcripts were observed during feeding of female ticks. Whereby a number of transcripts were upregulated during early feeding, presumably for feeding site establishment and then during late feeding, 52\% of these were downregulated, indicating that transcripts were required at specific feeding stages. This suggested that secretory proteins are under stringent transcriptional regulation that fine-tunes their expression in salivary glands during feeding. No open reading frames were predicted for 7947 transcripts. This class represented 17\% of the differentially expressed transcripts, suggesting a potential transcriptional regulatory function of long non-coding RNA in tick blood-feeding.

(Continued on next page)
\end{abstract}

\footnotetext{
*Correspondence: mansb@arc.agric.za

${ }^{1}$ Epidemiology, Parasites and Vectors, Onderstepoort Veterinary Research,

Agricultural Research Council, Onderstepoort, South Africa

${ }^{3}$ College of Agriculture and Environmental Sciences, University of South

Africa, Johannesburg, South Africa

Full list of author information is available at the end of the article
} 
(Continued from previous page)

Conclusions: The assembled sialotranscriptome greatly expands the sequence availability of $R$. zambeziensis, assists in our understanding of the transcription of secretory proteins during blood-feeding and will be a valuable resource for future vaccine candidate selection.

Keywords: Rhipicephalus zambeziensis, De novo transcriptome assembly, Tick salivary glands, Sialotranscriptomics, Secretory proteins, Next generation sequencing, Differential expression

\section{Background}

Ticks are hematophagous ectoparasites of a wide range of wild and domestic animals and humans worldwide, and serve as coincidental vectors of a variety of diseases $[1,2]$. After mosquitos, ticks are the second most important arthropod vector of human diseases and transmit more bacterial, viral and protozoan pathogens than any other arthropod [3]. Chemical acaricides have always been the most effective mechanism of tick control [4], but the emergence of acaricide-resistance [5] and health concerns due to chemically residues in meat, milk and the environment [6] have shifted the focus of tick biologists to the development of recombinant anti-tick vaccines [7-10]. The prospect of immunising animals by exposure to tick antigens was already acknowledged in the 1970's when extracted antigens from the tick gut were injected into guinea pigs and cattle, resulting in protection from subsequent tick challenges [11]. Since then a large number of recombinant tick proteins have been investigated and tested for protection efficiencies in hosts, with some showing very promising efficacy values (reviewed in [10]). Even though recombinant anti-tick vaccines have been shown to be attractive for tick control, their practical development has proven difficult as only two anti-tick vaccines have been commercialised to date, both targeting the same $R$. microplus Bm86 gut protein $[12,13]$. The search for effective vaccine candidates therefore continues.

Ticks have evolved a complicated cocktail of secretory proteins and molecules that are secreted into the host to evade host immune surveillance and suppress host defence responses, enabling ticks to feed unnoticed until repletion (reviewed in [14-20]). In recent years, it has been realised that tick salivary glands, the organs in closest proximity to the host during feeding and actively producing secretory proteins, are much more diverse than initially anticipated (reviewed in $[15,21]$ ). Previous studies have shown that secretory proteins are under positive selection $[22,23]$ and have been subjected to gene duplications, resulting in lineage-specific expansions [14-16, 24] and large multi-genic functionally redundant protein families [19, 21]. Large secretory protein families containing functionally redundant members confine vaccine development, where immunisation against a protein in a multi-genic family may be circumvented by the expression of another functionally redundant member in the same family [7]. It is therefore unlikely that secretory proteins will serve as successful targets for vaccine development in the future without a thorough understanding of the secretory protein families involved in blood-feeding and their temporal expression and regulation during feeding phases. Complete sequence datasets of ticks are required to address these caveats. The first completed genome sequence of a tick species, Ixodes scapularis, has recently become available from the collaborative effort of a global tick consortium [25, 26]. The large estimated genome sizes of hard tick species, 2.0-7.1 Gbp [27, 28], make whole genome sequencing an unlikely technology to be routinely adopted by tick biologists in the near future. A more realistic strategy for ticks is sequencing of the expressed RNA species in the salivary glands, generating tick sialotranscriptomes, of which a number have become available in recent years [29-39]. The availability of these transcriptomes affords unprecedented insight into tick salivary gland biology and the extensive expansion in secretory protein families. With the transcriptome sequencing of only a handful of the nearly 900 known tick species [1], it can be expected that the knowledge of tick salivary glands will greatly expand in the coming years.

Rhipicephalus zambeziensis is distributed through eastern and southern Africa and vectors the protozoan parasite Theileria parva, causative agent of East Coast fever, Corridor disease and January disease [40-42]. The main vector of T. parva, $R$. appendiculatus, has a much wider distribution throughout central, eastern and southern Africa [42, 43]. Rhipicephalus zambeziensis is better adapted to extreme environmental conditions [44] and naturally occurs in hotter and drier regions than $R$. appendiculatus [40]. One of the most pertinent differences between $R$. zambeziensis and $R$. appendiculatus is the variability in vector competence of T. parva. During infection experiments, more $R$. zambeziensis ticks were infected and at higher infection loads than $R$. appendiculatus ticks [45-47], indicating, at least experimentally, that $R$. zambeziensis is a better vector of T. parva. Furthermore, changes in climatic conditions (e.g. temperature and rainfall) can result in suitable environments in regions not normally part of the natural distribution of a tick species [48]. Due to the projected temperature increase and 
rainfall decrease in sub-Saharan Africa it has been predicted that environmental regions suitable for $R$. appendiculatus habitation will change [49], resulting in regions better suited for $R$. zambeziensis ticks that prefer hotter and drier conditions [50]. In a similar situation, the competent vector and invasive species $R$. microplus displaces $R$. decoloratus on a wide scale following climatic gradients, resulting in serious tickborne disease control issues [51]. The risk associated with the expansion of $R$. zambeziensis, a highly competent vector of $T$. parva, to a wider distribution due to projected climatic changes, may therefore have serious implications for the control of East Coast fever, Corridor disease and January disease.

Prior to the start of this study, only $33 R$. zambeziensis sequences (nucleotide and protein) were publically available. To alleviate the sequence shortcomings of $R$. zambeziensis, a representative, high quality sialotranscriptome was assembled de novo and annotated from female to male ticks during different feeding stages. The assembled transcriptome was used as reference to determine abundances and differential expression of transcripts and protein families during feeding and between different sexes, with special focus on transcripts predicted to be coding for secretory proteins. To our knowledge this is the first report of a de novo transcriptome of $R$. zambeziensis using next generation sequencing. This transcriptome will likely prove invaluable for future comparative studies analysing multi-genic secretory protein phylogenies and to broaden our understanding of tick biology and the secretory proteins involved in bloodfeeding and host immune modulation. Further, the transcriptome can be used for proteomic analyses in $R$. zambeziensis and for the future selection of potential vaccine candidates.

\section{Methods}

\section{Tick feeding and salivary gland dissection}

A parasite-free $R$. zambeziensis tick colony is maintained under standard laboratory and tick-rearing protocols at Onderstepoort Veterinary Research Institute [52]. The colony was initiated from ticks collected from vegetation in the Marakele National Park, Limpopo Province, South Africa, in 2010. Adult ticks were fed in feeding bags on disease-free Hereford (Bos taurus) cattle and carefully removed at 3 and 5 days after attachment. Unfed ticks, from the same batch of engorged nymphs as the fed ticks, were also processed. Twenty male and twenty female ticks were removed at each time point. Ticks were dissected directly after removal from the bovine and the salivary glands extracted and stabilised in RNAlater (Qiagen, Valencia, CA, USA) according to the manufacturer's specifications until RNA extraction.
RNA extraction, library preparation and sequencing Total RNA was extracted using the RNeasy Protect Mini Kit (Qiagen) and residual genomic DNA removed by DNase I digestion (Qiagen). Approximately $2 \mu \mathrm{g}$ of total RNA was used for library generation in the TruSeq RNA Sample Preparation kit (Illumina, San Diego, CA, USA). Fragment fractions of \pm 300 bp were excised after agarose gel electrophoreses to prepare the libraries for HiScanSQ $100 \mathrm{bp}$ paired end sequencing. Additionally, a pooled library from an equimolar mixture of all six samples was generated for MiSeq sequencing. This library was prepared from longer RNA fragments by a shortened RNA fragmentation step (changed from 8 to $3 \mathrm{~min}$ ) in the TruSeq Sample Preparation kit, according to the manufacturer's specifications. A high molecular weight library fraction ( \pm 600-1000 bp) was excised for subsequent MiSeq sequencing, generating 300 bp paired end reads. Sequencing was performed on the HiScanSQ and MiSeq Illumina instruments at the Biotechnology Platform Sequencing Facility (Agricultural Research Council, South Africa).

\section{Read quality filtering, de novo transcriptome assembly and evaluation}

Trimmomatic version 0.32 [53] was used to remove Illumina adaptor sequences and low quality bases from the sequence reads using the parameters; ILLUMINACLIP: TruSeq3-PE-2.fa: 2:30:10 LEADING:20 TRAILING:20 SLIDINGWINDOW:4:20 AVGQUAL:15 MINLEN:50. The SLIDINGWINDOW parameter was adjusted to 7:15 for MiSeq generated sequences. Following quality filtering, paired end MiSeq sequences that overlapped by at least 20 bases, were merged into a single sequence using the CLC Genomics Workbench version 7.5.1 (Qiagen). All quality-filtered and merged reads were pooled together to assemble a single de novo transcriptome of $R$. zambeziensis using the Trinity software package version 2.1.1 [54,55]. Standard k-mer size of 25 was used and a minimum k-mer coverage of two was selected to reduce the incorporation of potential sequencing errors. An expression level threshold of fragments per kilobase of exon per million fragments mapped (FPKM) value of 1 was used to select against lowly expressed transcripts, that possibly represent assembly artefacts or background expression [56-58]. The quality of the assembled transcriptome was evaluated using the Transrate version 1.0.0 software package [59] that estimates the quality of each transcript based on the mapping coverage of sequence reads and the alignment to a closely related reference sequence. The set of wellcurated predicted proteins from the sequenced tick genome, I. scapularis (IscaW1.4) [26], was used as reference. The Transrate transcript evaluation was used as an additional transcript selection process to remove 
low quality transcripts and improve the confidence in the final transcriptome.

\section{Transcriptome annotation}

The transcriptome was BLASTx aligned (E-value $<$ E-05) against the following protein databases: the National Center for Biotechnology Information (NCBI) nonredundant (NR) and Transcriptome Shotgun Assembly (TSA-NR) databases (retrieved April 2016), the UniProt Knowledgebases (UniProtKB/TrEMBL and UniProtKB/ Swiss-Prot, retrieved July 2016), the I. scapularis predicted peptides (IscaW1.4, retrieved July 2016) [26], an Acari database (AcariDB; containing all available mite and tick sequences, as described in [37]) retrieved July 2016, and the EuKaryotic Orthologous Groups (KOG) dataset, retrieved July 2016 [60]. Additionally, the transcriptome was BLASTn searched against the NCBI non-redundant nucleotide $(\mathrm{Nt})$ database (retrieved April 2016). The Blast2GO software package [61] was used to search the NR BLASTx results for Gene Ontology (GO) terms and visualised by the Web Gene Ontology Annotation Plot (WEGO) [62]. Kyoto Encyclopedia of Genes and Genomes (KEGG) orthology (KO) identifiers from the I. scapularis genome were assigned to the transcripts using the KEGG Automatic Annotation Server (KAAS) [63]. The protein-coding potential of the transcripts were determined by three software packages: Predictor of lncRNAs and mRNAs based on k-mer scheme v1.2 (PLEK) [64]; Coding Potential Calculator (CPC) [65]; and Coding-Potential Assessment Tool (CPAT) [66].

\section{Open reading frame prediction and annotation}

Translation frames obtained from transcripts with significant BLASTx searches against AcariDB were used to predict putative open reading frames (ORFs) of $240 \mathrm{bp}$ or longer using the OrfPredictor Server [67]. A second round of prediction for transcripts where ORFs were not predicted was performed using the orffinder.pl script [68]. The predicted ORFs were translated into amino acid sequences, BLASTp searched against the AcariDB database and compared to the BLASTx results of the transcripts. In cases where the BLAST results were not similar the ORFs were manually inspected and, where possible, ORFs were predicted using the Expert Protein Analysis System (ExPASy) Translate tool [69]. Predicted proteins were BLASTp searched against the search databases mentioned above as well as against NCBI's Conserved Domain Database (CDD) [70] and the Pfam database [71]. SignalP 4.1 [72] and Phobius [73] were used to identify putative signal peptide signatures and TMHMM 2.0 [74], to predict the transmembrane topology of the amino acid sequences. ORFs with no BLASTp or domain-based matches were discarded, after which CD-HIT v4.5.4 [75] was used to obtain a final non-redundant set of predicted proteins. These proteins were annotated in a priority order of: AcariDB, NR, UniProtKB/TrEMBL, I. scapularis proteins, Pfam database and CDD database. The AcariDB was used to classify the proteins into four main classes: putative secretory, housekeeping, unknown function and no hit proteins (proteins for which no significant matches were found in the database). The final set of predicted proteins was evaluated for completeness by searching for the presence of a conserved set of 1066 arthropod single-copy orthologous proteins using the Benchmarking Universal Single-Copy Orthologs (BUSCO) v2 software [76]. To evaluate whether the methodology pipeline followed in this study produced a representative set of Rhipicephalus salivary gland proteins, the predicted $R$. zambeziensis proteins were compared to the predicted proteins of two closely related tick sialotranscriptomes: $R$. pulchellus (NCBI Bioproject PRJNA170743) [34] and $R$. appendiculatus (NCBI Bioproject PRJNA297811) [37]. As a further validation of the assembled transcriptome and predicted proteins, 17 previously characterised $R$. appendiculatus protein sequences [77-84] were used in a BLASTp database to identify putative representatives in the $R$. zambeziensis transcriptome.

\section{Differential expression analysis}

The de novo assembled transcriptome of $R$. zambeziensis was used as reference for differential expression analysis, whereby the quality-filtered sequence reads of each time point was analysed against it separately. Additionally, the filtered reads within each sex were combined to perform differential expression analysis between male and female ticks. The Trinity software package contains custom scripts to facilitate differential expression analysis [54, 55]. Initially, the reads were mapped to the transcriptome using Bowtie 2 v2.2.3 [85], followed by abundance estimation with the RNA-Seq by Expectation-Maximization (RSEM) v1.2.31 software package [86] using the trimmed mean of $M$ values (TMM) normalisation [87]. The transcripts per million (TPM) values were determined and used to estimate the relative abundance of a transcript, also referred to as its expression level [88]. Differential expression analysis was performed using the Bioconductor/Empirical analysis of digital gene expression data in R (edgeR) v3.14.0 software package [89], using the fixed dispersion value of 0.4 (as recommended for biologically non-replicated samples), fold change of $>4$ and false discovery rate (FDR) $P$-value of $<0.01$. Chi-square tests with Bonferroni corrections were used for significance testing in comparisons of protein family expression. 


\section{Results}

De novo assembly and validation of the $R$. zambeziensis transcriptome

To assemble the salivary gland transcriptome of R. zambeziensis, between 22 and 37 million paired HiScanSQ sequencing reads were generated for each time point, together with about 22 million paired MiSeq reads from a pool of all time points (Additional file 1: Table S1). After adapter trimming and quality filtering, $81 \%$ of the HiScanSQ reads were retained in a paired end format and $7 \%$ as single ends. Of the MiSeq reads, $31 \%$ were retained as paired and $35 \%$ as single ends reads. All quality-filtered reads were combined to create a dataset containing approximately 192 million read 1 and 146 million read 2 sequencing reads, which was used for de novo assembly of the $R$. zambeziensis transcriptome. In total, 140,703 transcripts were assembled that were filtered based on expression level (FPKM values > 1) and read mapping confidences (as estimated by the transcriptome evaluation software, Transrate, [59]). This resulted in a final transcriptome of 23,631 high confidence transcripts (Table 1). Of the sequenced reads, 94\% mapped to the initial and $91 \%$ to the final transcriptome. This indicated that the nearly 120,000 transcripts excluded from the final assembly represented less than $3 \%$ of the total reads, likely representing mostly low quality transcripts. Furthermore, $79 \%$ of the transcripts in the final assembly were near full-length, showing a larger than $75 \%$ alignment coverage to their best BLAST matches in the I. scapularis protein set. The proteins predicted from the final transcriptome were analysed for assembly and annotation completeness by the BUSCO software package [76]. Eighty-six percent of the conserved proteins were both present and full-length and only $1.7 \%$ of the proteins were fragmented and not assembled in full-length copies. These metrics indicated that a high quality $R$. zambeziensis transcriptome was assembled, which was close to completion, mostly fulllength and highly representative of the sequence reads from which it was build.

\section{Annotation and characterisation of the $R$. zambeziensis transcriptome and predicted proteins}

The assembled $R$. zambeziensis transcriptome was annotated by sequence similarity searches against a number of databases, which resulted in 18,311 transcripts (78\% of the transcriptome) obtaining a significant match $(E$-value $<$ E-05) to at least one of the databases (Table 1, complete transcript annotation can be found in Additional file 2: Table S6). Nearly half of the transcripts were assigned Gene Ontology terms, which included 12,805 cellular components, 20,458 biological processes and 14,603 molecular functions (Additional file 3: Figure S1). Based on KOG functional categories,
Table 1 Summary of the R. zambeziensis transcriptome assembly and annotation statistics

\begin{tabular}{|c|c|}
\hline Transcriptome statistics & Value $^{\mathrm{a}}$ \\
\hline \multicolumn{2}{|l|}{ Transcriptome assembly statistics } \\
\hline Total number of transcripts & 23,631 \\
\hline Number of transcripts $>500 \mathrm{bp}$ & 19,903 \\
\hline Number of transcripts $>1 \mathrm{~kb}$ & 13,330 \\
\hline Number of transcripts $>10 \mathrm{~kb}$ & 80 \\
\hline Shortest transcript length (bp) & 201 \\
\hline Longest transcript length (bp) & 17,108 \\
\hline Mean length of transcripts (bp) & 1793.5 \\
\hline Median length of transcripts (bp) & 1193 \\
\hline Transcript N50 (bp) & 2807 \\
\hline Total bases in assembly (Mb) & 42.4 \\
\hline Ambiguous base calls (Ns) & 0 \\
\hline GC content (\%) & 49 \\
\hline Number of non-redundant predicted proteins & 13,584 \\
\hline \multicolumn{2}{|l|}{ Transcriptome annotation statistics $^{\mathrm{b}}$} \\
\hline BLASTx against NR & $12,756(54.0 \%)$ \\
\hline BLASTx against UniProtKB/TrEMBL & $16,451(69.6 \%)$ \\
\hline BLASTx against UniProtKB/Swiss-Prot & $10,572(44.7 \%)$ \\
\hline BLASTx against TSA-NR & $16,711(70.7 \%)$ \\
\hline BLASTx against Ixodes scapularis predicted peptides & $11,804(50.0 \%)$ \\
\hline BLASTx against EuKaryotic Orthologous Groups (KOG) & $9620(40.7 \%)$ \\
\hline BLASTx against AcariDB & $18,245(77.2 \%)$ \\
\hline Assigned with Gene Ontology $(\mathrm{GO})$ terms ${ }^{c}$ & $11,360(48.1 \%)$ \\
\hline Assigned with Enzyme Commission (EC) numbers ${ }^{c}$ & $3493(14.8 \%)$ \\
\hline Assigned with KEGG orthology $(\mathrm{KO})$ identifiers $^{\mathrm{d}}$ & $4869(20.6 \%)$ \\
\hline Annotated in at least one database & $18,311(77.5 \%)$ \\
\hline \multicolumn{2}{|c|}{$\begin{array}{l}\text { "Value indicating either the number of transcripts, proteins or bases, the } \\
\text { transcript length or percentage, as indicated in the table } \\
\text { bNumber (and \%) of transcripts annotated based on significant matches (E-value } \\
<\text { E-05) against databases as detailed in the Methods section } \\
{ }^{\mathrm{c} G O} \text { terms and EC numbers assigned with Blast2GO } \\
{ }^{\mathrm{d}} \text { From the KEGG (Kyoto Encyclopedia of Genes and Genomes) Automatic } \\
\text { Annotation Server (KAAS) using the I. scapularis genome }\end{array}$} \\
\hline
\end{tabular}

most $R$. zambeziensis transcripts were classified as "Post translational modification, protein turnover, chaperones", "General function prediction only" or "Signal transduction mechanisms" (Additional file 3: Figure S2). The KEGG pathway analysis revealed that most transcripts belonged to the "Ribosome", "RNA transport" and "Spliceosome" pathways (Additional file 3: Figure S3). Additionally, alignment against the NCBI Nt database retrieved full-length copies of all 4 ribosomal RNA molecules in the $R$. zambeziensis transcriptome (Additional file 2: Table S6).

In total, 15,737 open reading frames (ORFs) were predicted from $66 \%(15,684)$ of the $R$. zambeziensis transcripts. A small fraction of the transcripts (0.2\%) encoded for more than one ORF. The predicted ORFs 
were translated into amino acid (aa) sequences and reduced, based on $100 \%$ aa sequence similarity, to a nonredundant set of 13,584 predicted proteins (predicted protein annotation can be found in Additional file 4: Table S7). Eighty-six percent of these were estimated to be putatively full-length proteins as they contained both a predicted start and stop codon. Of the transcripts for which no ORFs could be predicted (7947 transcripts), $98 \%$ were assigned as putative non-coding sequences by at least two coding potential databases (Additional file 2: Table S6). Signal peptide signatures were observed in 3488 predicted proteins, 2169 proteins contained transmembrane domains and 8061 proteins showed similarity to at least a single Pfam domain (the 30 most abundant R. zambeziensis Pfam domains can be found in Additional file 3: Figure S4). AcariDB was used to classify the predicted proteins into: 8139 housekeeping, 2569 secretory, 1706 unknown function and 1170 no hit proteins. Protein annotation was performed based on a priority database order, as set out in the Methods section. Finally, based on the invertebrate mitochondrial genetic code, full-length gene copies of all $13 R$. zambeziensis mitochondrial proteins were predicted and annotated (Additional file 4: Table S7).

\section{Comparison of the $R$. zambeziensis transcriptome to other Rhipicephalus sialotranscriptomes}

The predicted proteins from the assembled $R$. zambeziensis transcriptome were compared to publically available transcriptomes of two closely related Rhipicephalus species, for which protein predictions were available. The number of predicted $R$. zambeziensis proteins $(13,584)$ were similar to the number of predicted proteins in the $R$. appendiculatus $(12,761)$ and $R$. pulchellus $(11,227)$ transcriptomes. The lengths of the $R$. zambeziensis proteins (57-4928 aa, average 341 aa) were slightly shorter than those predicted for $R$. appendiculatus (70-4966 aa, average 400 aa) and R. pulchellus (666645 aa, average 471 aa). However, from the length distribution of the predicted proteins it was evident that the $R$. zambeziensis transcriptome was enriched for shorter proteins without compromising the distribution of longer proteins (Fig. 1a). Pfam domain prediction and comparison among the species showed that most of the domains (64\%) were shared among all the transcriptomes (Fig. 1b). More domains were shared between the phylogenetically closer species, $R$. zambeziensis and $R$. appendiculatus (15\%), than $R$. pulchellus and any of the two species (about $5 \%$ shared with each species). These results showed that the assembled $R$. zambeziensis transcriptome and resulting predicted proteins were comparable to published tick sialotranscriptomes and therefore representative of proteins expected in the salivary glands of feeding Rhipicephalus ticks.
Search for putative functional orthologues in the $R$. zambeziensis transcriptome

To verify that the assembled $R$. zambeziensis transcriptome could be used as a resource in future, we searched for the presence of representatives of 17 previously characterised $R$. appendiculatus proteins. A number of functional studies have been performed in $R$. appendiculatus, and based on its close relation to $R$. zambeziensis, it was chosen as target for the homology searches. Representative $R$. zambeziensis sequences were identified for $13 R$. appendiculatus proteins based on more than $70 \%$ protein identity to the target sequences (Additional file 1: Table S2). Nine putative orthologues were obtained when the minimum protein identity was increased to $90 \%$, representing the identification of a putative orthologous sequence for more than half (53\%) of the targeted sequences. These included the: Immunoglobulin Gbinding proteins A-C (IGBP-MA-C) [77]; Femalespecific histamine-binding protein 1 (HBP1) [78]; $R$. appendiculatus serine proteinase inhibitor serpins 1-3 (RAS-1-3) [81]; Rhipicephalus immuno-dominant molecule 36 (RIM36) [79]; and Japanin-like-RA1 precursor (JL-RA1) [84]. Four R. zambeziensis proteins shared less than $70 \%$ protein identity to their best match, indicating either that these proteins were not assembled in the $R$. zambeziensis transcriptome, that $R$. zambeziensis does not contain orthologues of these proteins or that protein divergence in these families changed the sequences considerably. It is of importance to mention that actual protein function can only be determined by functional protein characterisation, which remains to be performed for the $R$. zambeziensis proteins. Then, except for RIM36 that was assembled in two separate nonoverlapping transcripts and RAS-2 that contained no predicted stop codon, all the other $R$. zambeziensis proteins contained both a predicted start and stop codon and were predicted to be full-length. This indicated that the $R$. zambeziensis transcriptome is a valuable resource from which full-length sequences of potential vaccine candidates can be selected in future.

\section{Expression composition in the feeding phases and sexes of the $R$. zambeziensis transcriptome}

Between 19 and 30 million clean, paired sequence reads were obtained for each time point after quality filtering (Additional file 1: Table S1) and mapped to the assembled transcriptome of $R$. zambeziensis to estimate transcript abundance. Suitable mapping rates of around 90\% were achieved for each time point. Overall, a wide expression range of $0.4-46,919$ TPM was observed in the $R$. zambeziensis transcriptome and only a few transcripts (560 transcripts, $2.4 \%$ of the transcriptome) accounted for $80 \%$ of the total expression (Additional file 2: Table S6). Two thirds of the assembled transcripts were predicted to 


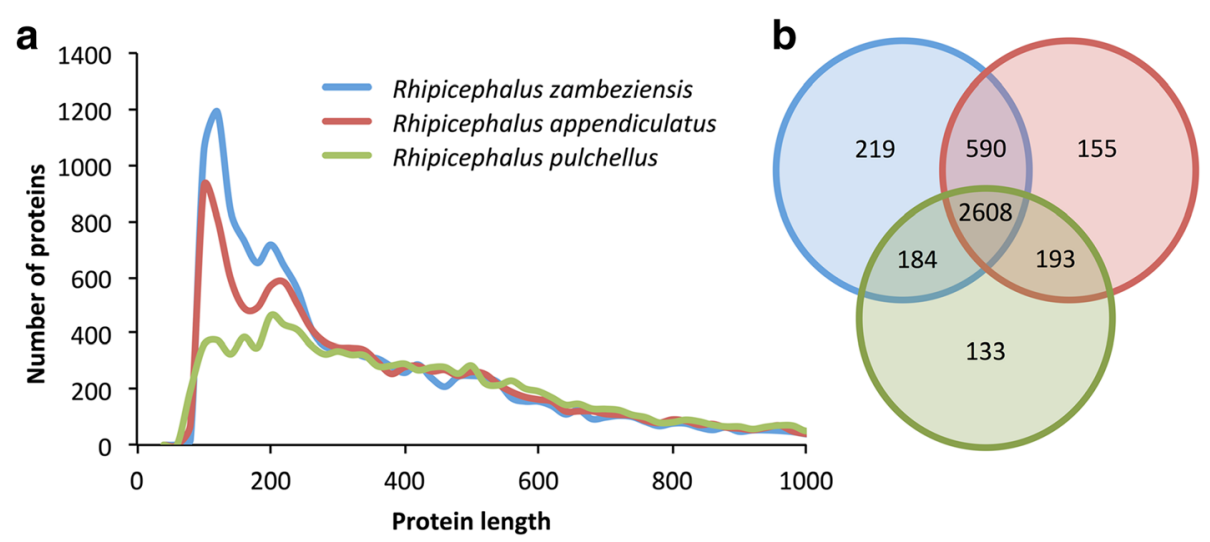

Fig. 1 Comparisons of the predicted $R$. zambeziensis proteins to proteins of two closely related Rhipicephalus species. a Length distribution of the predicted proteins of $R$. zambeziensis, R. appendiculatus and R. pulchellus. The number of proteins is indicated based on a protein length sliding window of 20 amino acids (aa), showing a maximum length of 1000 aa. b Pfam domain comparison of the three Rhipicephalus species. Datasets used: 13,584 predicted proteins from the assembled R. zambeziensis transcriptome, 12,761 R. appendiculatus proteins [37] and 11,227 R. pulchellus proteins [34]. Blue represents R. zambeziensis; red, R. appendiculatus; and green, R. pulchellus

be protein-coding and represented $84 \%$ of the expression in the transcriptome (Fig. 2a). Only a small fraction of the predicted proteins were classified as secretory proteins (19\%; 2569 proteins), but this protein class represented more than half of the transcription in the coding fraction of the salivary glands (52\%, Fig. $2 b$ ). Conversely, the largest protein class at $60 \%$, the housekeeping proteins, represented only $36 \%$ of the transcript expression. Within the secretory protein class, $71 \%$ of the expression was as a result of a single protein family, the Glycine rich superfamily (Table 2). Some of the other large contributors to the secretory protein class were the Lipocalin (5.6\%), Secretory - unknown function (5.0\%) and Bovine pancreatic trypsin inhibitor (2.5\%) families. Further examination of the secretory protein families showed that their expression composition changed greatly over time, resulting in unique secretory protein compositions for each time point in female and male ticks (Fig. 3a, b, Additional file 1: Table S3). The Glycine rich superfamily was the most abundant family in 4 of the 6 time points, where it ranged from 67 to $84 \%$ of the expression, reaching a maximum contribution to

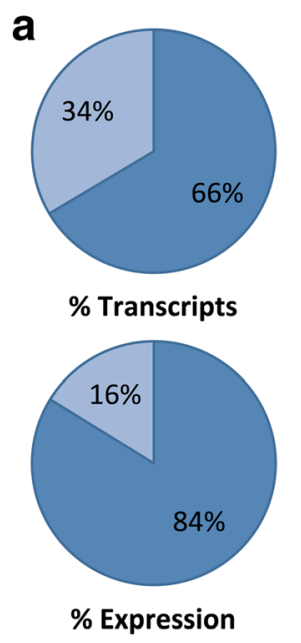

$\square$ Protein coding $\square$ Non protein coding
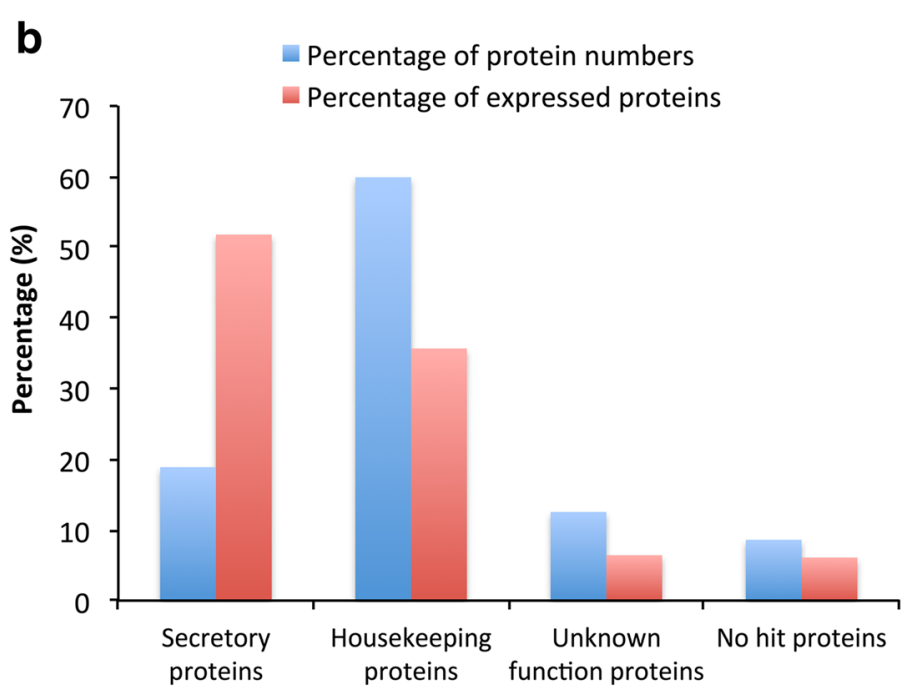

Protein Classes

Fig. 2 Classification and expression analysis in the R. zambeziensis transcriptome. a Proportions of predicted protein-coding (indicated by dark blue colouring) and predicted non protein-coding (light blue) transcripts and their contribution to total expression. b Proportions of protein numbers (blue) and expression contribution (red) of the four predicted protein classes to the total protein-coding fraction of the transcriptome. Expression was estimated by transcripts per million (TPM) 
Table 2 Characterisation of the secretory protein families in the R. zambeziensis transcriptome

\begin{tabular}{|c|c|c|c|c|c|}
\hline Secretory protein family & $\begin{array}{l}\text { Number of family } \\
\text { members }\end{array}$ & $\begin{array}{l}\text { Proportion of family } \\
\text { members (\%) }\end{array}$ & $\begin{array}{l}\text { Average } \\
\text { TPM }^{\mathrm{a}} \text { value }\end{array}$ & $\begin{array}{l}\text { Sum of the family } \\
\text { expression }\left(T^{2} M^{2}\right)\end{array}$ & $\begin{array}{l}\text { Proportion of family } \\
\text { expression (\%) }\end{array}$ \\
\hline Lipocalin & 588 & 22.90 & 39.88 & $23,452.37$ & 5.62 \\
\hline Bovine pancreatic trypsin inhibitor & 307 & 11.95 & 34.07 & $10,458.51$ & 2.51 \\
\hline Reprolysin & 213 & 8.29 & 19.36 & 4123.57 & 0.99 \\
\hline TIL domain & 177 & 6.89 & 38.05 & 6735.14 & 1.61 \\
\hline Glycine rich superfamily & 161 & 6.27 & 1842.35 & $296,618.84$ & 71.07 \\
\hline Basic tail secreted protein & 111 & 4.32 & 43.04 & 4777.46 & 1.14 \\
\hline 8.9 kDa family & 91 & 3.54 & 74.27 & 6758.57 & 1.62 \\
\hline Digestive system (including Serine proteases) & 87 & 3.39 & 9.57 & 832.96 & 0.20 \\
\hline Mucin & 62 & 2.41 & 47.14 & 2922.86 & 0.70 \\
\hline 28 kDa Metastriate family & 56 & 2.18 & 31.67 & 1773.45 & 0.42 \\
\hline Evasin & 55 & 2.14 & 33.33 & 1832.92 & 0.44 \\
\hline Secretory - unknown function & 46 & 1.79 & 455.62 & $20,958.56$ & 5.02 \\
\hline $\begin{array}{l}\text { Folding, sorting and degradation } \\
\text { (including Cathepsins) }\end{array}$ & 46 & 1.79 & 88.22 & 4058.19 & 0.97 \\
\hline Cystatin & 45 & 1.75 & 17.27 & 777.09 & 0.19 \\
\hline Gluzincin & 43 & 1.67 & 7.34 & 315.45 & 0.08 \\
\hline Serpin & 33 & 1.29 & 6.30 & 207.96 & 0.05 \\
\hline Ixodegrin B & 32 & 1.25 & 55.31 & 1770.02 & 0.42 \\
\hline One of each family & 28 & 1.09 & 3.71 & 103.68 & 0.02 \\
\hline Carboxypeptidase inhibitor & 27 & 1.05 & 16.01 & 432.37 & 0.10 \\
\hline Chitin-binding proteins & 26 & 1.01 & 20.30 & 527.89 & 0.13 \\
\hline 5'-Nucleotidase & 25 & 0.97 & 6.56 & 163.87 & 0.04 \\
\hline Transport and catabolism & 24 & 0.93 & 35.23 & 845.47 & 0.20 \\
\hline 24 kDa family & 21 & 0.82 & 24.99 & 524.87 & 0.13 \\
\hline $7 \mathrm{~dB}$ family & 19 & 0.74 & 11.34 & 215.53 & 0.05 \\
\hline DA-P36 family & 19 & 0.74 & 10.15 & 192.90 & 0.05 \\
\hline Defensin & 19 & 0.74 & 211.69 & 4022.20 & 0.96 \\
\hline ML domain & 17 & 0.66 & 417.39 & 7095.58 & 1.70 \\
\hline Antigen 5 family & 14 & 0.55 & 57.55 & 805.64 & 0.19 \\
\hline Microplusin & 14 & 0.55 & 47.61 & 666.59 & 0.16 \\
\hline 8 kDa Amblyomma family & 13 & 0.51 & 24.52 & 318.79 & 0.08 \\
\hline Sphingomyelinase & 11 & 0.43 & 2.93 & 32.22 & 0.01 \\
\hline Glycan biosynthesis and metabolism & 10 & 0.39 & 9.36 & 93.63 & 0.02 \\
\hline Lipid metabolism & 10 & 0.39 & 5.88 & 58.76 & 0.01 \\
\hline Transcription & 10 & 0.39 & 3.75 & 37.52 & 0.01 \\
\hline Translation & 8 & 0.31 & 10.62 & 84.92 & 0.02 \\
\hline Serine/threonine protein kinase & 8 & 0.31 & 6.59 & 52.70 & 0.01 \\
\hline Carbohydrate metabolism & 8 & 0.31 & 3.53 & 28.21 & 0.01 \\
\hline Thyropin & 7 & 0.27 & 22.67 & 158.66 & 0.04 \\
\hline Fibrinogen-related domain & 7 & 0.27 & 20.84 & 145.85 & 0.03 \\
\hline Glutathione metabolism & 7 & 0.27 & 16.63 & 116.41 & 0.03 \\
\hline Metalloprotease & 7 & 0.27 & 8.28 & 57.93 & 0.01 \\
\hline Dermacentor 9 kDa expansion & 6 & 0.23 & 11.81 & 70.86 & 0.02 \\
\hline Replication and repair & 6 & 0.23 & 5.56 & 33.37 & 0.01 \\
\hline
\end{tabular}


Table 2 Characterisation of the secretory protein families in the R. zambeziensis transcriptome (Continued)

\begin{tabular}{|c|c|c|c|c|c|}
\hline Secretory protein family & $\begin{array}{l}\text { Number of family } \\
\text { members }\end{array}$ & $\begin{array}{l}\text { Proportion of family } \\
\text { members (\%) }\end{array}$ & $\begin{array}{l}\text { Average } \\
\text { TPM }^{a} \text { value }\end{array}$ & $\begin{array}{l}\text { Sum of the family } \\
\text { expression }\left(T^{2} M^{2}\right)\end{array}$ & $\begin{array}{l}\text { Proportion of family } \\
\text { expression (\%) }\end{array}$ \\
\hline Immunoglobulin G binding protein A & 5 & 0.19 & 989.88 & 4949.38 & 1.19 \\
\hline Phospholipase A2 & 5 & 0.19 & 9.54 & 47.69 & 0.01 \\
\hline Kazal/vWf domain & 4 & 0.16 & 4.78 & 19.11 & 0.005 \\
\hline Hirudin & 3 & 0.12 & 108.72 & 326.17 & 0.08 \\
\hline SALP15/Ixostatin & 3 & 0.12 & 35.45 & 106.35 & 0.03 \\
\hline 14 kDa family & 3 & 0.12 & 9.75 & 29.25 & 0.01 \\
\hline Kazal domain & 3 & 0.12 & 9.28 & 27.85 & 0.01 \\
\hline Signal transduction & 3 & 0.12 & 2.23 & 6.68 & 0.002 \\
\hline Madanin & 2 & 0.08 & 153.53 & 307.05 & 0.07 \\
\hline Energy metabolism & 2 & 0.08 & 13.13 & 26.25 & 0.01 \\
\hline CDIV & 2 & 0.08 & 9.44 & 18.88 & 0.005 \\
\hline EF hand domain & 2 & 0.08 & 5.33 & 10.65 & 0.003 \\
\hline Histamine release factor & 1 & 0.04 & 6057.33 & 6057.33 & 1.45 \\
\hline Fatty acid-binding protein & 1 & 0.04 & 70.19 & 70.19 & 0.02 \\
\hline Kazal/SPARC domain & 1 & 0.04 & 70.11 & 70.11 & 0.02 \\
\hline Immune system & 1 & 0.04 & 21.85 & 21.85 & 0.01 \\
\hline Cysteine rich hydrophobic domain 2 & 1 & 0.04 & 10.83 & 10.83 & 0.003 \\
\hline 26 kDa family & 1 & 0.04 & 5.49 & 5.49 & 0.001 \\
\hline Proline rich & 1 & 0.04 & 5.09 & 5.09 & 0.001 \\
\hline Cysteine rich & 1 & 0.04 & 2.23 & 2.23 & 0.001 \\
\hline
\end{tabular}

${ }^{\text {aTPM }}$ (transcripts per million) values were used to estimate expression

expression at the third day of feeding in each sex. In unfed females the most predominant family was the Histamine release factor (HRF) family (38\%) and in day 5 fed females, Lipocalin was the most abundant (26\%, Fig. 3a). Notably, the HRF family that contributed largely to the expression in the salivary glands of unfed female and male ticks consisted of only a single family member (Rzam_Mc198). At a TPM value of 6057 , this transcript was the 20th highest expressed transcript in the $R$. zambeziensis transcriptome.

\section{Differential expression in the $R$. zambeziensis salivary glands} Pairwise comparisons between time points within each sex identified 2832 significantly differentially expressed transcripts, of which 1927 were specific to the feeding phases of female ticks, 663 were male feeding-specific and 242 were shared between the feeding phases of the sexes. A between-gender comparison was performed by combining the reads of all the time points in each sex prior to mapping and expression analysis. This resulted in 1209 differentially expressed transcripts, of which 641 were upregulated in females and 568, in males. Half of the differentially expressed transcripts $(1470 ; 49 \%)$ were classified as belonging to the secretory protein class. The remaining 572 (19\%), 350 (11\%), 113 (4\%) and 510 (17\%) transcripts belonged to the housekeeping, unknown function, no hit or no ORF classes, respectively (Fig. 4a).
The secretory protein families with the largest number of differentially expressed transcripts were the Lipocalin (342 transcripts; 23\% of the differentially expressed transcripts in the secretory protein class), Bovine pancreatic trypsin inhibitor (184; 13\%), Reprolysin (134; 9\%), and Glycine rich $(93 ; 7 \%)$ families. Remarkably, $57 \%$ of the transcripts classified as secretory proteins were differentially expressed in the salivary glands of $R$. zambeziensis (Fig. 4b), indicating that ticks use a large repertoire of secretory proteins during feeding. Much smaller proportions, 6-21\%, of the other classes showed variation in expression and in total $13 \%$ of the assembled transcripts were differentially expressed. Furthermore, differentially expressed transcripts accounted for a substantial contribution (45\%) of the total expression observed in the salivary glands. This was more pronounced in the secretory protein class, where $72 \%$ of the expression was as a result of differentially expressed transcripts. This suggested that the variation observed in the expression composition of the secretory protein families during feeding (Fig. 3) is under strict transcriptional regulation that continually fine-tunes the expression in the salivary glands.

Of the differentially expressed transcripts identified in the between-gender comparison, 376 female and 259 male transcripts were classified as belonging to the secretory protein class (Additional file 1: Table S4). 


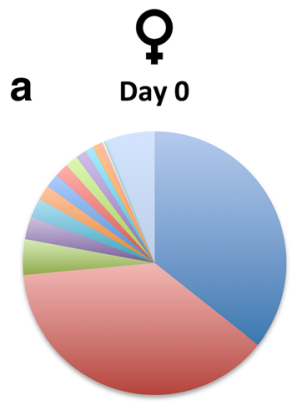

Day 3

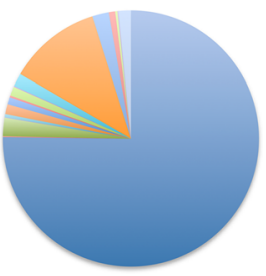

Day 5

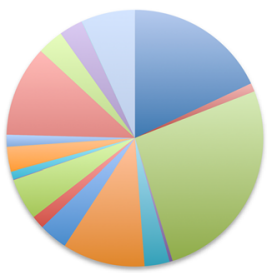

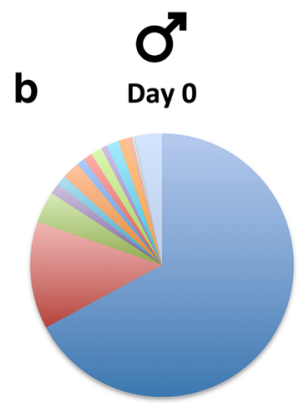

Day 3

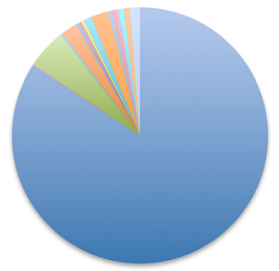

Day 5

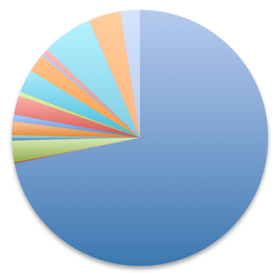

- Glycine rich superfamily

Histamine release factor

- Lipocalin

- Transport and catabolism

Mucin

= Bovine pancreatic trypsin inhibitor

- Reprolysin

= Folding, sorting and degradation

- Basic tail secreted protein

$=24 \mathrm{kDa}$ family

- TIL domain

- Secretory - unknown function

Defensin

I $8.9 \mathrm{kDa}$ family

Ixodegrin B

= $28 \mathrm{kDa}$ Metastriate family

- ML domain

- Immunoglobulin $\mathrm{G}$ binding protein $\mathrm{A}$

Other secretory proteins

Fig. 3 Expression proportions of the R. zambeziensis secretory protein families during feeding. The proportions of the highest contributing secretory protein families of female (a) and male (b) ticks at different feeding time points are indicated. Expression levels were measured by transcripts per million (TPM). Colour key representing the protein families is indicated. Expression values can be found in Additional file 1: Table S3
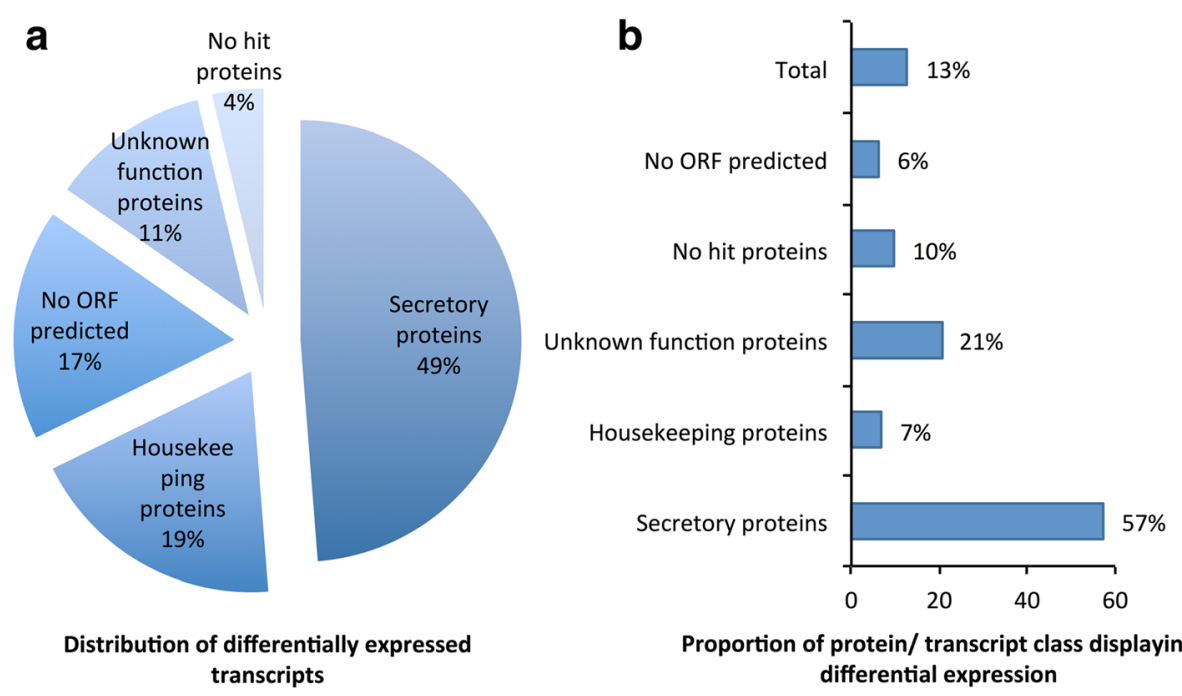

Proportion of protein/ transcript class displaying differential expression

Fig. 4 Overview of differential expression in the R. zambeziensis sialotranscriptome. a Classification of differentially expressed transcripts into different protein or transcript classes. $\mathbf{b}$ Proportion of differential expression observed within each protein or transcript class. Differential expression analyses were performed using the edgeR software package (with the parameters: fixed dispersion $=0.4$, fold change $>4$ and FDR $P<0.01$ ) 
Significantly more transcripts of the Lipocalin (140 female vs 42 male transcripts), $8.9 \mathrm{kDa}$ (36 vs 12), Reprolysin (27 vs 3) and $28 \mathrm{kDa}$ Metastriate (23 vs 2) families were upregulated in females after implementing a Bonferroni corrected $P<0.0013$. In males, the TIL (Trypsin Inhibitor-like) domain ( 4 vs 29), Folding, sorting and degradation (including Cathepsins; 1 vs 17), Digestive system (including Serine proteases; 0 vs 41), Cystatin (0 vs 26) and $7 \mathrm{~dB}(0$ vs 13) families showed significantly more upregulated transcripts when compared to females. Of these, the Digestive system, Cystatin and $7 \mathrm{~dB}$ families exhibited male-specific upregulation, as no members of these families were upregulated in females. A number of other secretory families showed large differences between the sexes and even gender-specific upregulation, albeit not significant after Bonferroni correction.

\section{Dynamic expression patterns of the $R$. zambeziensis secretory protein families}

Many transcripts belonging to secretory protein families were significantly upregulated during the early tick feeding phase (from unfed to day 3 fed ticks), in both female (541 transcripts) and male (335) ticks (Fig. 5a, b,
Additional file 1: Table S5). When considering late feeding (feeding progression from day 3 to 5), in specifically females, most of the differentially expressed secretory transcripts were downregulated (Fig. 5c). Surprisingly, about $52 \%$ of these downregulated transcripts were the same transcripts that were upregulated during early feeding, implying that certain secretory transcripts were only required during early feeding stages in females. Hardly any significant differential expression was observed in the male late feeding phase (Fig. $5 \mathrm{~d}$ ), demonstrating that the male day 3 and 5 time points were highly similar in their secretory family expression. Comparisons between unfed and day 5 fed female and male ticks revealed a large number of upregulated secretory transcripts in both sexes, together with some downregulated transcripts in females (Fig. 5e, f). Only about $42 \%$ of these upregulated transcripts in female ticks were shared with the transcripts upregulated during early feeding (day 0-3), likely due to more than half of the transcripts upregulated in early feeding undergoing downregulation in female late feeding. Conversely, in males, due to the few significant expression differences observed between day 3 and 5, a larger overlap of $74 \%$

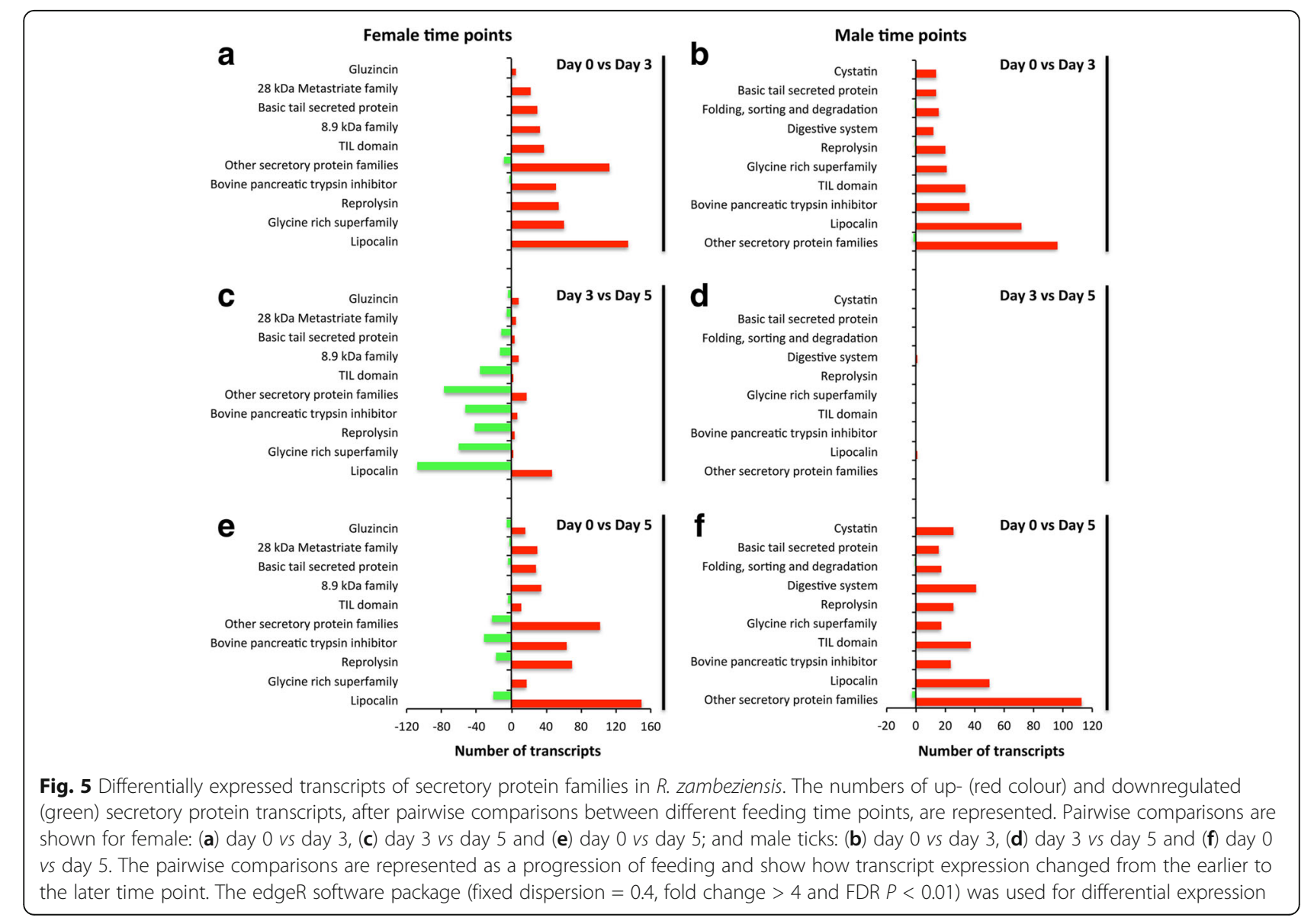


was seen between the upregulated transcripts in the day $0-3$ and day 0-5 comparisons. Non-significant expression differences between male day 3 and day 5, which would not be picked up by our analyses, might explain the differences observed in the upregulation profiles of the day $0-3$ and day $0-5$ comparisons in males (Fig. 5b, f). Furthermore, comparisons between the number of upregulated transcripts in each secretory family between day 0 3 and day $0-5$ revealed that significantly more transcripts of the Glycine rich (61 vs 17 transcripts, $\chi^{2}=24.82, d f=1$, $P<0.0001)$ and TIL domain (38vs $11, \chi^{2}=14.88, d f=1$, $P=0.00011)$ families were upregulated in earlier feeding points in females (Fig. 5a, e, Additional file 1: Table S5). Also, significantly more of the Digestive system (including Serine proteases, 12 vs 41, $\chi^{2}=15.87, d f=1, P<0.0001$ ) transcripts were upregulated in later feeding points in males (Fig. 5b, f). These results suggested that in tick salivary glands, genes of the secretory protein class are temporally regulated to alter the protein cocktail that is secreted into the host as feeding progresses.

\section{Discussion}

The main aim of this study was the de novo assembly, annotation and characterisation of the sialotranscriptome of $R$. zambeziensis, a vector of T. parva. Thus far, $R$. zambeziensis has been a largely neglected tick species due to its limited distribution through eastern and southern Africa [40], which was reflected by the small number of publically available sequences of $R$. zambeziensis prior to the start of this study; only 31 nucleotide and 2 protein sequences available in GenBank. In the present study this shortfall was alleviated by the transcriptome assembly and deposition of 13,584 annotated predicted proteins of $R$. zambeziensis into the public domain. This set of predicted proteins is a valuable resource for future vaccine candidate selection in $R$. zambeziensis, as was shown by the availability of mostly full-length versions of previously characterised proteins. The $R$. zambeziensis transcriptome was constructed from sequence reads from male and female ticks, unfed and representative phases of early and late feeding ticks, to represent a large proportion of genes involved in adult tick feeding. Great care was taken to assemble a high quality $R$. zambeziensis transcriptome that was representative of the reads from which it was assembled, near complete, containing a large proportion of fulllength protein sequences and similar to closely related tick sialotranscriptomes of $R$. pulchellus [34] and $R$. appendiculatus [37]. Indeed, the number of predicted protein sequences in the $R$. zambeziensis transcriptome was similar to the 11,105 genes predicted to be expressed in the salivary glands of $I$. scapularis, the only tick with a completely sequenced genome $[25,26]$. Provisional functions were assigned to the assembled $R$. zambeziensis transcriptome such as histamine [78] and immunoglobulin [77] binding, serine proteinase inhibition [81] and immunomodulation [84]. These functions have been predicted based on high protein identity $(\geq 90 \%)$ to previously characterised $R$. appendiculatus proteins, but will require functional studies to be confirmed in $R$. zambeziensis. Furthermore, the predicted $R$. zambeziensis proteins were annotated and $19 \%$ were classified as putative secretory proteins, in accordance with $13-37 \%$ annotated in previously assembled tick sialotranscriptomes [23, 29-31, 33, $34,37-39]$. In total, 8139 of the predicted proteins were classified as putative housekeeping proteins, similar to the number of proteins identified in other metastriate transcriptomes $[29,31,37]$ and the predicted number of core housekeeping genes in Chelicerata species, approximately 7000 [20]. Full-length sequences of all 13 mitochondrial genes and all four rRNA molecules were also assembled and can be used in future phylogenetic analyses.

The sequence reads of each time point were independently mapped to the assembled transcriptome to estimate transcript abundance during feeding. During RNA extraction, all ticks in a time point were pooled to obtain enough tissue for extraction and sequencing, similar to methodology followed in other tick transcriptomic studies $[23,30,32,33,35,36,90]$. The edgeR software package can accommodate non-replicated samples using strict parameters [89], resulting in the identification of fewer false positives but also fewer differentially expressed transcripts. Using these stringent parameters, only transcripts differing by more than a 29 -fold change were assigned as differentially expressed in the $R$. zambeziensis transcriptome. Consequently, only large expression differences were considered significant within this work, resulting in high confidence in the assigned differentially expressed transcripts but also the possibility that important feeding genes might have been missed if they had smaller variable expression levels. Nevertheless, 13\% of the assembled transcripts showed differential expression and these accounted for nearly half (45\%) of the expression in the salivary glands.

In this work, we were mainly interested in the transcriptional response of secretory proteins to tick feeding and we therefore investigated them in greater detail. While the secretory protein class represented only 19\% of the predicted proteins in the $R$. zambeziensis salivary glands, this class accounted for the majority of transcript expression (52\%). Similar transcript abundance of a small proportion of secretory proteins was seen in other tick sialotranscriptomes: 17 and $63 \%$ of the proportions of, respectively, predicted proteins and expression were classified as secretory proteins in $R$. appendiculatus [37]; 23 and $62 \%$ in Hyalomma excavatum [39]; and 37 and 49\% in Amblyomma americanum [33]. Nearly half (49\%) of the differentially expressed transcripts in $R$. zambeziensis were classified as secretory proteins. Previous 
studies have also shown that the majority of differentially expressed transcripts in tick salivary glands are classified as secretory proteins $[23,33]$. The abundance of transcripts coding for secretory proteins in tick salivary glands was not unexpected as the salivary glands are in primary contact with the host into which secretory proteins are actively being secreted to escape host haemostasis, inflammation and immune response. Remarkably, most (57\%) of the predicted $R$. zambeziensis secretory proteins showed significant differential transcript expression, even using the stringent parameters used in this study, and accounted for $72 \%$ of the expression observed in the secretory proteins class. These large variations in the $R$. zambeziensis secretory protein expression resulted in each time point resembling a unique transcriptome with different expression proportions of secretory protein families. These differences were more pronounced in female ticks that require more on-host time to feed to repletion. Similarly, changes in secretory protein family compositions during feeding were seen in the saliva of $R$. microplus [91] and I. scapularis [92].

Differential expression analysis of the R. zambeziensis secretory protein families revealed dynamic profiles of transcript expression during feeding of female ticks. Most of the differentially expressed secretory transcripts were upregulated during early feeding whereas during late feeding most were downregulated. A similar expression pattern was also observed in A. americanum salivary glands [93]. However, in R. zambeziensis $52 \%$ of the upregulated transcripts in early feeding were shared with the downregulated transcripts in late feeding, indicating that these transcripts were specifically required only during early feeding and the establishment of the feeding site in female ticks. Further assessment of the expression regulation in the most differentially expressed secretory protein families (Lipocalin, Bovine pancreatic trypsin inhibitor, Reprolysin and Glycine rich), revealed similar patterns of intricate transcript up- and downregulation and a trend towards preferential expression of transcripts in a single time point in female salivary glands (data not shown). Correspondingly, members of multi-gene secretory protein families showed remarkable differential expression in the salivary glands of $A$. americanum [33, 94] and I. ricinus [23], where certain transcripts were uniquely expressed during different developmental stages or feeding time points. Similar profiles were described in I. scapularis ticks based on proteomic analyses of saliva collected in 24-h intervals during feeding [92], indicating that transcriptomic profiles of salivary glands are transferable to the proteome being secreted into the host. These results suggest that members of tick secretory protein families are under tight transcriptional regulation that result in complex compositions of secretory proteins in different feeding phases, equipping ticks with the diversity in secretory proteins to evade host immune defences. Indeed, the authors of the above-mentioned studies refer to the expression dynamics observed in tick salivary glands as 'sialome switching' or a form of antigenic variation of secretory proteins to evade host immune recognition while still maintaining host immune modulation to feed successfully. Antigenic variation is a mechanism by which infecting microorganisms systematically alter or 'switch' their surface proteins to remain unnoticed by the host immune defences (reviewed in [95]). In ticks, antigenic variation will rely on functional redundancy of the multi-genic secretory protein families, where alternate members could be sequentially expressed to evade immune recognition but still retain the same function or a number of members could be expressed concurrently to result in an additive functional effect while maintaining low immunogenicity to each protein [21]. The ability of ticks to preserve crucial blood-feeding functionalities while simultaneously evading the host immune response by altering exposed antigens, make ticks remarkably well adapted to feed for extended periods of time on their hosts.

The above-mentioned conclusions were based on results generated from female ticks [23, 33, 92, 94]. Both female and male ticks were analysed in the current $R$. zambeziensis sialotranscriptome, showing that the expression profiles of secretory proteins were much less complex in males compared to females during feeding. Similar to the low expression variation in male $A$. americanum ticks during feeding [93], only five differentially expressed transcripts were observed between the third and fifth day of feeding of male $R$. zambeziensis ticks, indicating that the male sialotranscriptome necessary for successful feeding had mostly already been established by the third day of feeding. Furthermore, a number of transcripts were differentially expressed between female and male ticks, indicating that some secretory protein families were differentially regulated between the sexes and that female and male ticks feed and evade host immune defences using different mechanisms. This was similar to observations made before $[34,37,93,96]$. Male ticks feed by continuously attaching and deattaching from the host to be in close proximity to female ticks [3] in order to mate and assist with establishing a stable feeding cavity from where females can feed to repletion to complete their life-cycles. This might mean that male ticks need a constant supply of secretory proteins to be ready to feed at any time or to secrete decoy antigens into the host to assist females during feeding, which has been shown for male-specific Immunoglobulin-binding proteins [97]. Furthermore, the Serine protease, Cathepsin and Cystatin families, were found to be nearly exclusively upregulated in male $R$. zambeziensis salivary glands when compared to 
females, similar to families found upregulated in males in previous studies [34, 37]. During mating, male ticks salivate on their spermatophores before inserting them into the female genital pore using their mouthparts [98]. Proteases and protease inhibitors have abundantly been found in insect seminal fluid $[99,100]$ and the upregulation of their transcripts in male salivary glands might suggest an additional function for these secretory proteins in tick reproduction (as proposed by Tan et al. [34]).

In order to alter the exposed antigens presented to their hosts and to feed unnoticed until repletion, ticks must have evolved stringent temporal regulatory processes to sequentially express secretory proteins, of which the mechanism is still largely unknown. Adamson and colleagues [101] showed that SDS3, a component of the Sin3 histone deacetylase corepressor complex involved in histone modification and repression of transcription, was particularly downregulated concurrently with a number of expression differences in secretory protein transcripts in A. maculatum ticks. The authors proposed that tick secretory proteins might, to some degree, be under epigenetic regulation by histone modification and chromatin remodeling. Based on these results, 34 genes associated with histone modification were identified in the transcriptome of I. ricinus [23]. Additionally, recent surveillance of 5 histone and 34 histone modifying enzyme transcripts in I. scapularis indicated that Anaplasma phagocytophilum altered tick epigenetics to assist pathogen infection and multiplication during infection [102], which changed the expression of the tick's salivary transcripts [103]. The relationship between epigenetic gene regulation and long non-coding RNA (lncRNA) has been well established in recent years (as reviewed in [104-106]), by which lncRNAs bind to and act as scaffolds between specific sequences in the genome to be transcriptionally regulated and chromatinmodifying enzymes. Long ncRNAs are RNA molecules larger than $200 \mathrm{bp}$ that contain no open reading frames (> $300 \mathrm{bp}$ ) for protein translation [107]. These lncRNAs have been largely unexplored in ticks, although a set of 4439 predicted non-coding RNA genes were annotated in the recently completed I. scapularis genome [26]. Also, in $R$. appendiculatus, 7414 assembled transcripts contained no predicted ORFs and this set of transcripts represented a striking $40 \%$ of the differentially expressed transcripts in the salivary glands [37]. Similarly, in the current transcriptome assembly of $R$. zambeziensis, no open reading frames could be predicted for a third of the assembled transcripts (7947 transcripts). These transcripts represented $16 \%$ of the total expression in the salivary glands and most (98\%) showed low proteincoding potential [64-66]. These no ORF-containing transcripts also represented $17 \%$ (510 transcripts) of the differentially expressed transcripts in $R$. zambeziensis during feeding. This set of transcripts with no predicted ORFs likely contains putative lncRNAs, but also probably protein-coding transcripts for which the predicted ORFs were too small to be retained or potentially misassembled sequences of no biological significance. These protein-coding transcripts are unfortunately not easily distinguishable from lncRNAs and warrants further examination. The large number of differentially expressed transcripts in feeding $R$. zambeziensis ticks, for which no ORFs could be predicted, suggests that lncRNA molecules might be involved in tick blood-feeding, potentially through the transcriptional regulation of tick secretory proteins. This could either be as a complementary mechanism to the proposed epigenetic regulation by Adamson et al. [101] or by other more direct regulatory functions of lncRNAs, such as: acting as transcriptional co-regulators or co-repressors, binding to transcription factors to act as decoys, controlling alternative splice variants, stabilising mRNA by sequestering micro RNAs (miRNAs) away from targets, to name but a few (reviewed in [104, 106]).

The role of lncRNAs in the regulation of the vertebrate immune system has been well established in the last years (reviewed in $[108,109]$ ), although the function in arthropod immunity remains to be determined. Recently, a potential role for lncRNAs in vector immunity has been identified by the increase of a number of Aedes aegypti lncRNAs in response to virus and endosymbiont infection [110]. It has also been shown that the vertebrate immune system can be modulated by viral lncRNAs to enhance virus survival during infection of the host [111, 112]. Apart from normal secretory processes, tick salivary glands also excrete molecules into the saliva by a mechanism known as apocrine secretion, where whole pieces of the cells are shed and cytoplasmic contents excreted into the lumen of the acinus $[20,113,114]$. It is conceivable that should it be proven that ticks express lncRNAs that target host defences, this excretion mechanism might be the entry point of the lncRNAs into tick saliva. The functions of lncRNAs in arthropods are still elusive, but the significant number of differentially expressed no ORFcontaining transcripts observed in R. zambeziensis, the extensive transcriptional regulatory functions described for lncRNAs and the potential host immunomodulation by parasite-derived lncRNAs, warrant further investigations of these important RNA molecules in ticks.

Similar to observations in other Rhipicephalus species $[34,37]$, we found that the Glycine rich family in the transcriptome of $R$. zambeziensis was an exceptionally large contributor (71\%) to the total expression of the secretory protein class. Maruyama and colleagues [115] previously postulated that tick species with short mouthparts, e.g. Rhipicephalus, require large quantities of Glycine rich proteins to form the cement-cone for 
adhesion to the host's skin $[3,116]$. The conclusions of the authors [115] were based on only a few species and limited Expression Sequence Tag (EST) data, but recent advances in next generation sequencing of tick transcriptomes have extended the hypothesis to more species with better confidence. Accordingly, the expression contribution of the Glycine rich family to the secretory protein class in ticks with short mouthparts was between 48 and $71 \%$ based on three Rhipicephalus species ([34, 37], present study) and between 3 and 28\% for ticks with long mouthparts based on four Amblyomma [31, 33] and a single Hyalomma [39] species. Rhipicephalus $(0.34 \mathrm{~mm})$ has much shorter mouthparts than Hyalomma $(0.62 \mathrm{~mm})$ and Amblyomma $(1.27 \mathrm{~mm})$ ticks, with no overlap between the three groups [117]. Another observation in the $R$. zambeziensis transcriptome was that the Glycine rich family was expressed nearly twice as much in male than female ticks $(382,258$ TPM in males and 202,292 TPM in females), an observation shared with other Rhipicephalus species [34, 37]. A large contribution of Glycine rich proteins was observed in early feeding females, followed by downregulation in late feeding, as would be expected for the establishment of the cement-cone, which is generally created within the first three to 4 days after attachment [118]. Contrary, in male salivary glands, Glycine rich proteins remained disproportionally overrepresented in all time points. In light of the cement-cone, the abundance of the Glycine rich family in males is counterintuitive as one would expect female ticks, considering their extended feeding times and exceptional expansion in body size [3], to require a larger cement-cone, and therefore more Glycine rich expression. However, the Glycine rich family is a superfamily to which a number of alternative functions have been ascribed that might be involved during feeding and employed by male ticks to maintain the feeding site. For instance, some Glycine rich proteins have shown to function as antimicrobial peptides in insects (reviewed in [119]) and might assist prolonged tick feeding by keeping the feeding cavity free from microbial infections. Additionally, Glycine rich proteins may also contain RNA-recognition motifs to bind RNA during transcriptional regulation and splicing (reviewed in [120]) or to bind nucleic extrusions from neutrophil extracellular traps of the host defence response (as proposed by Maruyama et al. [115]). Furthermore, in ticks, some Glycine rich proteins have shown to be immunedominant $[79,121]$ and might be used by the males as decoy antigens to preoccupy the host defences, thereby assist female feeding [97].

In addition, the $R$. zambeziensis data revealed that even though the expression level of the Glycine rich family was double in males than females, the number of differentially expressed transcripts were considerably fewer: six male and 18 female differentially expressed transcripts for the gender-specific comparison; and 22 male and 89 female differentially expressed transcripts for the feeding-specific comparisons. Similarly, in R. pulchellus, two male and 19 female-specific upregulated transcripts were found [34]. These data suggest that females require a more diverse set of Glycine rich proteins in their salivary glands, potentially indicative of a more complex, longer lasting cement-cone that might facilitate the prolonged feeding times and extreme body size expansions seen in female ticks [3]. Alternatively, the Glycine rich protein diversity observed in female ticks might be a mechanism of antigenic variation. Antigenic variation has been proposed as the reason $R$. microplus, a one-host tick that feeds for an extensive amount of time on the same host, showed a larger variety of Glycine rich proteins when compared to two- or threehost ticks [115]. As a final consideration, the nucleotide sequences of the Glycine rich proteins are low in complexity and contain multiple repeats. A technical limitation of assembly algorithms when using short next generation sequencing data, such as Illumina sequences, is the difficulty to assemble low complexity and repeat sequences [122]. This becomes apparent when considering that from all the predicted $R$. zambeziensis proteins only $5 \%$ had no predicted methionine start codons, but when assessing only the predicted Glycine rich proteins, $35 \%$ had no predicted start codons, indicating a number of fragmented or incomplete sequences in this family. An example of this was seen by the inability to assembly the putative orthologue of RIM36, a Glycine rich cement protein of $R$. appendiculatus [79], into a single transcript. Third generation sequencing technologies, Pacific BioSciences Iso-Seq and Oxford Nanopore MinION sequencing, generate sequence reads with much longer lengths $(>10 \mathrm{~Kb})$ than Illumina sequencing and are used to improve general transcriptome assembly complications, e.g. repeat regions and alternative splice variants $[123,124]$. In future, the use of these technologies will assist with the assembly of full-length transcripts for the Glycine rich protein family and tick multi-gene families in general.

Another major contributing family to the transcriptome expression of specifically unfed ticks was the Histamine release factor (HRF) family, representing 38\% of female and $13 \%$ of male transcript expression in unfed ticks. This family consisted of a single protein, Rzam_Mc198, which showed $73.4 \%$ protein identity to the I. scapularis tick histamine release factor (tHRF, AAY66972.1) [125]. Tick HRF binds to basophil cells and induces the release of histamine $[125,126]$, a molecule integral to the vertebrate inflammatory response. Ticks are highly sensitive to histamine during the early stages of feeding, but the sensitivity diminishes towards 
the end of feeding [127] and histamine becomes required for rapid engorgement presumably by facilitating vascular permeability and blood flow [125]. Contrary to the increasing expression of $t H R F$ in I. scapularis as feeding progressed [125], a considerable overexpression of $t H R F$ was observed in unfed $R$. zambeziensis ticks. Knowing the detrimental effect of histamine on feeding site establishment [127], it is likely that tHRF has a different function prior to feeding, in the free-living stages of $R$. zambeziensis. Histamine release factor proteins are highly conserved and found in a number of eukaryotes and, apart from involvement in inflammation by histamine release, have functions in early developmental processes and cell survival by anti-apoptotic activity in response to stressors such as heat shock and oxidative stress (reviewed in [128]). Desiccation, due to low environmental humidity and high temperatures, is one of the main constraints of free-living ticks surviving the many months between feeding stages, resulting in ticks requiring adaptations to survive in certain climatic regions [129]. Rhipicephalus zambeziensis naturally occurs in hot, dry regions with low humidity in southern Africa $[40,50]$ and has shown high tolerance for extreme experimental abiotic conditions, including low humidity and high temperature [44]. Additionally, the marked wet and dry climatic seasons in southern Africa results in an extended period of inactivity of $R$. zambeziensis where only few of the life stages are found on hosts in the long, dry season [50]. Yet, the tick does not undergo behavioural diapause [130] and it is unclear how it survives these long periods. It is conceivable that the overexpression of $t H R F$ in unfed $R$. zambeziensis might contribute to the survival of the tick during freeliving and questing periods, potentially by means of increased tolerance to stressors such as water/moisture deficiencies, an association that remains to be experimentally verified.

Another function of tHRF was seen during pathogen transmission, where the I. scapularis $t H R F$ gene was upregulated during Borrelia burgdorferi-infection. Silencing of the gene by RNA interference or immunisation with recombinant tHRF resulted in reduced transmission rates to the vertebrate host [125], indicating that tHRF functions as a saliva-assisted transmission (SAT) molecule [131]. Rhipicephalus zambeziensis and $R$. appendiculatus are vectors of the protozoan parasite, T. parva [41, 42], and it has experimentally been shown that $R$. zambeziensis has better vector competence for T. parva [45-47]. Integral to the life-cycle of T. parva is the tick's salivary glands where the pathogen multiplies and awaits transmission to the vertebrate host [132] and important vector-pathogen interactions can be expected to occur in these organs. Indeed, the overall level of $t H R F$ expression in the salivary glands of $R$. zambeziensis, at 6057
TPM, was about 5-times more than the expression seen for the R. appendiculatus tHRF gene, 1212 TPM, generated in a previous study [37]. As tHRF has been shown to function as a SAT molecule enhancing pathogen transmission, the variation in expression between the tHRFs of $R$. zambeziensis and $R$. appendiculatus might, at least to some degree, explain the variation observed in the vector competence of $T$. parva of the two tick species.

\section{Conclusions}

In this work we reported the first de novo transcriptome assembly of $R$. zambeziensis. The deposition of 13,584 annotated predicted proteins into GenBank vastly improves the sequence availability of the tick and will assist future studies in this largely neglected tick species. As would be expected, an abundance of secretory protein transcription was seen in the salivary glands, the organs in close proximity to the host that are actively expressing proteins to orchestrate host immune modulation. A large number of these secretory transcripts showed significant differential expression resulting in intricate expression profiles that changed considerably over feeding stages. Dynamic secretory protein transcription is possibly a result of antigenic variation, a mechanism previously proposed by which exposed tick antigens are 'switched' while evading host immune detection. Interchangeable expression of secretory proteins will have serious implications for recombinant vaccine development, as multi-genic secretory proteins have shown to be functionally redundant. Tick sialotranscriptomes, such as $R$. zambeziensis, will assist recombinant vaccine development by having the sequences of all members of a multi-gene family available to design vaccines against potentially shared antigens. Furthermore, a large number of transcripts for which no ORFs could be predicted were differentially expressed in the $R$. zambeziensis transcriptome, suggesting a role for lncRNAs in tick bloodfeeding and specifically, transcriptional regulation of secretory proteins, and motivates further investigation of this potentially important RNA species in ticks. Additionally, the transcriptome will enhance our understanding of tick biology, for example, in this study we observed: data suggesting gender differences in cementcone complexity and feeding behaviour; a potential candidate gene for survival of free-living $R$. zambeziensis ticks; and a potential molecular explanation for the differences observed in vector competence between two tick species. Understanding the biology of $R$. zambeziensis is of major importance for tick-borne disease control in southern Africa, as the tick is an efficient vector of $T$. parva, well adapted for extreme environments and, with the intensification of climate change, predicted to expand its natural distribution in future. 


\section{Additional files}

Additional file 1: Table S1. Specifications of $R$. zambeziensis library preparation procedures and the size and number of sequence reads before and after quality filtering. Table S2. Putative R. zambeziensis orthologues of previously characterised $R$. appendiculatus proteins. Table S3. Expression proportions of the highest contributing secretory protein families during different feeding time points. Table S4. Differential expression analysis between female and male $R$. zambeziensis ticks. Table S5. Number of differentially expressed transcripts in the protein classes and secretory protein families of $R$. zambeziensis during feeding. (DOCX $80 \mathrm{~kb}$ )

Additional file 2: Table S6. Annotation of the $R$. zambeziensis transcriptome. (XLSX $11156 \mathrm{~kb})$

Additional file 3: Figure S1. Gene Ontology (GO) characterisation of the $R$. zambeziensis transcriptome. Level $2 \mathrm{GO}$ terms of cellular components, molecular functions and biological processes were visualised using WEGO (Web Gene Ontology Annotation Plot). These included 18,436 cellular components, 20,487 biological processes and 9659 molecular functions. Figure S2. KOG clustering of R. zambeziensis transcripts. In total, $9620 R$. zambeziensis transcripts were assigned to 25 Eukaryotic Clusters of Orthologs (KOG) functional categories, of which 3814 were unique KOG terms. Figure S3. Top 30 most abundant KEGG pathways identified in the $R$. zambeziensis transcriptome. Four thousand eight hundred and sixty nine transcripts were assigned to 338 I. scapularis Kyoto Encyclopedia of Genes and Genomes (KEGG) pathways. Figure S4. Top 30 Pfam domain occurrences in the $R$. zambeziensis predicted proteins. A total of 13,451 Pfam domains were observed in the $R$. zambeziensis proteins, of which 3601 were unique. Eight thousand and sixty one of the proteins contained at least one Pfam domain. (DOCX $553 \mathrm{~kb}$ )

Additional file 4: Table S7. Annotation of the predicted proteins of $R$. zambeziensis. (XLSX 7708 kb)

\section{Acknowledgements}

Dr. Vinet Coetzee of the Department of Genetics, University of Pretoria, South Africa is thanked for editorial comments on the manuscript.

\section{Funding}

This work was supported by the Economic Competitive Support Programme (30/01/N010) and the National Research Foundation (NRF) Incentive Funding for Rated Researchers (NRF-Mans). MD was supported by an NRF/Department of Science and Technology - Professional Development Program (NRF/DST-PDP) studentship. The funding bodies had no role in study design, data collection, analysis and interpretation, decision to publish, or preparation of the manuscript.

\section{Availability of data and materials}

The datasets supporting the conclusions of this article are included within the article and its additional files. Sequence data supporting the findings of this study have been deposited in public sequence databases. Raw sequence reads have been deposited in the NCBI Short Read Archive (SRA, SRR5438376-82) under Bioproject accession number PRJNA381085 and are available from http:// www.ncbi.nlm.nih.gov/Traces/sra/. The transcript sequences have been deposited in the NCBI Transcriptome Shotgun Assembly (TSA) project under accession number GFPF00000000 and are available from https://www.ncbi.nlm.nih.gov/ Traces/wgs/. The transcriptome version described in this paper is the first version, GFPF01000000

\section{Authors' contributions}

MHD and BJM conceived and designed the study and BJM supplied the funding. DD, RP and BJM were responsible for tick colony maintenance, tick feeding and dissections. MHD performed the experimental work, acquired the data and performed the bioinformatic analyses. MHD and BJM interpreted the data and drafted the manuscript. MHD, DD, RP, DJGR and BJM revised the manuscript. All authors read and approved the final manuscript.

\section{Ethics approval}

Ethical approval was obtained from the OVI Animal Ethics Committee (approval number: AEC12.11, extended to AEC01.15) and the College of Agriculture and Environmental Sciences Animal Ethics Review Committee of the University of South Africa (approval number: 2014/CAES/098).
Consent for publication

Not applicable.

\section{Competing interests}

The authors declare that they have no competing interests.

\section{Publisher's Note}

Springer Nature remains neutral with regard to jurisdictional claims in published maps and institutional affiliations.

\section{Author details}

${ }^{1}$ Epidemiology, Parasites and Vectors, Onderstepoort Veterinary Research, Agricultural Research Council, Onderstepoort, South Africa. ${ }^{2}$ Biotechnology Platform, Agricultural Research Council, Onderstepoort, South Africa. ${ }^{3}$ College of Agriculture and Environmental Sciences, University of South Africa, Johannesburg, South Africa. ${ }^{4}$ Department of Veterinary Tropical Diseases, Faculty of Veterinary Science, University of Pretoria, Pretoria, South Africa.

Received: 9 May 2017 Accepted: 27 July 2017

Published online: 10 August 2017

\section{References}

1. Jongejan F, Uilenberg G. The global importance of ticks. Parasitology. 2004; 129(Suppl):S3-S14.

2. Dennis DT, Piesman JF. Overview of tick-borne infections of humans. In: Goodman JL, Dennis DT, Sonenshine DE, editors. Tick-borne diseases of humans. Washington, DC: American Society of Microbiology Press; 2005. p. 3-11.

3. Sonenshine DE. Biology of ticks. Volume 1. Oxford: Oxford University Press; 1991

4. Willadsen P. Tick control: thoughts on a research agenda. Vet Parasitol. 2006;138:161-8

5. Abbas RZ, Zaman MA, Colwell DD, Gilleard J, lqbal Z. Acaricide resistance in cattle ticks and approaches to its management: the state of play. Vet Parasitol. 2014;203:6-20.

6. Graf JF, Gogolewski R, Leach-Bing N, Sabatini GA, Molento MB, Bordin EL, et al. Tick control: an industry point of view. Parasitology. 2004; 129(Suppl.):S427-S42.

7. Guerrero FD, Miller RJ, Pérez de León AA. Cattle tick vaccines: many candidate antigens, but will a commercially viable product emerge? Int J Parasitol. 2012;42:421-7.

8. Marcelino I, de Almeida AM, Ventosa M, Pruneau L, Meyer DF, Martinez D, et al. Tick-borne diseases in cattle: applications of proteomics to develop new generation vaccines. J Proteome. 2012;75:4232-50.

9. de la Fuente J, Kopáček P, Lew-Tabor A, Maritz-Olivier C. Strategies for new and improved vaccines against ticks and tick-borne diseases. Parasite Immunol. 2016:38:754-69.

10. Lew-Tabor AE, Rodriguez-Valle M. A review of reverse vaccinology approaches for the development of vaccines against ticks and tick borne diseases. Ticks Tick Borne Dis. 2016;7:573-85.

11. Allen JR, Humphreys SJ. Immunisation of guinea pigs and cattle against ticks. Nature. 1979;280:491-3.

12. Willadsen P, Bird P, Cobon GS, Hungerford J. Commercialisation of a recombinant vaccine against Boophilus microplus. Parasitology. 1995; 110(Suppl):S43-50.

13. Canales M, Enríquez A, Ramos E, Cabrera D, Dandie H, Soto A, et al. Largescale production in Pichia pastoris of the recombinant vaccine $\mathrm{Gavac}^{\mathrm{TM}}$ against cattle tick. Vaccine. 1997;15:414-22.

14. Mans BJ, Neitz AWH. Adaptation of ticks to a blood-feeding environment: evolution from a functional perspective. Insect Biochem Mol Biol. 2004;34:1-17.

15. Francischetti IMB, Sá-Nunes A, Mans BJ, Santos IM, Ribeiro JMC. The role of saliva in tick feeding. Front Biosci. 2009;14:2051-88.

16. Mans BJ. Evolution of vertebrate hemostatic and inflammatory control mechanisms in blood-feeding arthropods. J Innate Immun. 2011:3:41-51.

17. Oliveira CJF, Sá-Nunes A, Francischetti IMB, Carregaro V, Anatriello E, Silva JS, et al. Deconstructing tick saliva: non-protein molecules with potent immunomodulatory properties. J Biol Chem. 2011;286:10960-9.

18. Kotál J, Langhansová H, Lieskovská J, Andersen JF, Francischetti IMB, Chavakis T, et al. Modulation of host immunity by tick saliva. J Proteome. 2015;128:58-68. 
19. Mans BJ. Glandular matrices and secretions: blood-feeding arthropods. In: Cohen E, Moussian B, editors. Extracellular composite matrices in arthropods. Switzerland: Springer; 2016. p. 625-88.

20. Mans BJ, de Castro MH, Pienaar R, de Klerk D, Gaven P, Genu S, et al. Ancestral reconstruction of tick lineages. Ticks Tick Borne Dis. 2016;7: 509-35.

21. Chmelař J, Kotál J, Kopecký J, Pedra JHF, Kotsyfakis M. All for one and one for all on the tick-host battlefield. Trends Parasitol. 2016;32:368-77.

22. Dai S-X, Zhang A-D, Huang J-F. Evolution, expansion and expression of the Kunitz/BPTI gene family associated with long-term blood feeding in Ixodes scapularis. BMC Evol Biol. 2012;12:4.

23. Kotsyfakis M, Schwarz A, Erhart J, Ribeiro JMC. Tissue-and time-dependent transcription in Ixodes ricinus salivary glands and midguts when blood feeding on the vertebrate host. Sci Rep. 2015;5:9103.

24. Mans BJ, Andersen JF, Francischetti IMB, Valenzuela JG, Schwan TG, Pham VM, et al. Comparative sialomics between hard and soft ticks: implications for the evolution of blood-feeding behavior. Insect Biochem Mol Biol. 2008; 38:42-58.

25. de la Fuente J, Waterhouse RM, Sonenshine DE, Roe RM, Ribeiro JMC, Sattelle DB, et al. Tick genome assembled: new opportunities for research on tick-host-pathogen interactions. Front Cell Infect Microbiol. 2016;6:103.

26. Gulia-Nuss M, Nuss AB, Meyer JM, Sonenshine DE, Roe RM, Waterhouse RM, et al. Genomic insights into the Ixodes scapularis tick vector of Lyme disease. Nat Commun. 2016;7:10507.

27. Ullmann AJ, Lima CMR, Guerrero FD, Piesman J, Black WCT. Genome size and organization in the blacklegged tick, Ixodes scapularis and the southern cattle tick, Boophilus microplus. Insect Mol Biol. 2005;14:217-22.

28. Geraci NS, Johnston JS, Robinson JP, Wikel SK, Hill CA. Variation in genome size of argasid and ixodid ticks. Insect Biochem Mol Biol. 2007;37:399-408.

29. Karim S, Singh P, Ribeiro JMC. A deep insight into the sialotranscriptome of the gulf coast tick, Amblyomma maculatum. PLoS One. 2011;6:e28525.

30. Schwarz A, von Reumont BM, Erhart J, Chagas AC, Ribeiro JMC, Kotsyfakis M. De novo Ixodes ricinus salivary gland transcriptome analysis using two nextgeneration sequencing methodologies. FASEB J. 2013;27:4745-56.

31. Garcia GR, Gardinassi LG, Ribeiro JMC, Anatriello E, Ferreira BR, Moreira HNS, et al. The sialotranscriptome of Amblyomma triste, Amblyomma parvum and Amblyomma cajennense ticks, uncovered by 454-based RNA-seq. Parasit Vectors. 2014:7:430.

32. Mudenda L, Pierlé SA, Turse JE, Scoles GA, Purvine SO, Nicora CD, et al. Proteomics informed by transcriptomics identifies novel secreted proteins in Dermacentor andersoni saliva. Int J Parasitol. 2014;44:1029-37.

33. Karim S, Ribeiro JMC. An insight into the sialome of the lone star tick, Amblyomma americanum, with a glimpse on its time dependent gene expression. PLoS One. 2015;10:e0131292.

34. Tan AWL, Francischetti IMB, Slovak M, Kini RM, Ribeiro JMC. Sexual differences in the sialomes of the zebra tick, Rhipicephalus pulchellus. J Proteome. 2015:117:120-44

35. Xu X-L, Cheng T-Y, Yang H, Yan F, Yang Y. De novo sequencing, assembly and analysis of salivary gland transcriptome of Haemaphysalis flava and identification of sialoprotein genes. Infect Genet Evol. 2015;32:135-42.

36. Yu X, Gong H, Zhou Y, Zhang H, Cao J, Zhou J. Differential sialotranscriptomes of unfed and fed Rhipicephalus haemaphysaloides, with particular regard to differentially expressed genes of cysteine proteases. Parasit Vectors. 2015;8:597.

37. de Castro MH, De Klerk D, Pienaar R, Latif AA, Rees DJG, Mans BJ. De novo assembly and annotation of the salivary gland transcriptome of Rhipicephalus appendiculatus male and female ticks during blood feeding. Ticks Tick Borne Dis. 2016;7:536-48.

38. Ong CT, Rodriguez-Valle M, Moolhuijzen PM, Barrero RA, Hunter A, Szabo T, et al. Exploring the transcriptomic data of the Australian paralysis tick, Ixodes holocyclus. GSTF J Vet Sci. 2016;3:1.

39. Ribeiro JMC, Slovák M, Francischetti IMB. An insight into the sialome of Hyalomma excavatum. Ticks Tick Borne Dis. 2017;8:201-7.

40. Walker JB, Norval RAI, Corwin MD. Rhipicephalus zambeziensis sp. Nov., a new tick from eastern and southern Africa, together with a redescription of Rhipicephalus appendiculatus Neumann, 1901 (Acarina, Ixodidae). Onderstepoort J Vet Res. 1981;48:87-104.

41. Lawrence JA, Norval RAI, Uilenberg G. Rhipicephalus zambeziensis as a vector of bovine theileriae. Trop Anim Health Prod. 1983;15:39-42.

42. Norval RAl, Perry BD, Young AS. The epidemiology of theileriosis in Africa. London: Academic Press; 1992.
43. Walker AR, Bouattour A, Camicas JL, Estrada-Peña A, Horak IG, Latif AA, et al. Ticks of domestic animals in Africa: a guide to identification of species. Bioscience Reports: Edinburgh; 2003.

44. Madder M, Speybroeck N, Bilounga A, Helleputte D, Berkvens D. Survival of unfed Rhipicephalus appendiculatus and Rhipicephalus zambeziensis adults. Med Vet Entomol. 2005;19:245-50.

45. Potgieter FT, Stoltsz WH, Blouin EF, Roos JA. Corridor disease in South Africa: a review of the current status. J S Afr Vet Assoc. 1988;59:155-60.

46. Blouin EF, Stoltsz WH. Comparative infection rates of Theileria parva lawrencei in salivary glands of Rhipicephalus appendiculatus and Rhipicephalus zambeziensis. Onderstepoort J Vet Res. 1989;54:211-3.

47. Ochanda H, Young AS, Medley GF, Perry BD. Vector competence of 7 rhipicephalid tick stocks in transmitting 2 Theileria parva parasite stocks from Kenya and Zimbabwe. Parasitology. 1998;116:539-45.

48. Dantas-Torres F. Climate change, biodiversity, ticks and tick-borne diseases: the butterfly effect. Int J Parasitol Parasites Wildl. 2015;4:452-61.

49. Olwoch JM, Reyers B, Engelbrecht FA, Erasmus BFN. Climate change and the tick-borne disease, Theileriosis (East Coast fever) in sub-Saharan Africa. J Arid Environ. 2008:72:108-20.

50. Norval RAI, Walker JB, Colborne J. The ecology of Rhipicephalus zambeziensis and Rhipicephalus appendiculatus (Acarina, Ixodidae) with particular reference to Zimbabwe. Onderstepoort J Vet Res. 1982;49:181-90.

51. Lynen G, Zeman P, Bakuname C, Di Giulio G, Mtui P, Sanka P, et al. Shifts in the distributional ranges of Boophilus ticks in Tanzania: evidence that a parapatric boundary between Boophilus microplus and B. decoloratus follows climate gradients. Exp Appl Acarol. 2008;44:147-64.

52. Heyne H, Elliott EGR, Bezuidenhout JD. Rearing and infection techniques for Amblyomma species to be used in heartwater transmission experiments. Onderstepoort J Vet Res. 1987;54:461-71.

53. Bolger AM, Lohse M, Usadel B. Trimmomatic: a flexible trimmer for Illumina sequence data. Bioinformatics. 2014;30:2114-20.

54. Grabherr MG, Haas BJ, Yassour M, Levin JZ, Thompson DA, Amit I, et al. Full-length transcriptome assembly from RNA-Seq data without a reference genome. Nat Biotechnol. 2011;29:644-52.

55. Haas BJ, Papanicolaou A, Yassour M, Grabherr MG, Blood PD, Bowden J, et al. De novo transcript sequence reconstruction from RNA-Seq: reference generation and analysis with trinity. Nat Protoc. 2013;8:1494-512.

56. Mortazavi A, Williams BA, McCue K, Schaeffer L, Wold B. Mapping and quantifying mammalian transcriptomes by RNA-Seq. Nat Methods. 2008; 5:621-8.

57. Gan Q, Chepelev I, Wei G, Tarayrah L, Cui K, Zhao K, et al. Dynamic regulation of alternative splicing and chromatin structure in Drosophila gonads revealed by RNA-seq. Cell Res. 2010;20:763-83.

58. Hebenstreit D, Fang M, Gu M, Charoensawan V, van Oudenaarden A, Teichmann SA. RNA sequencing reveals two major classes of gene expression levels in metazoan cells. Mol Syst Biol. 2011;7:497.

59. Smith-Unna R, Boursnell C, Patro R, Hibberd JM, Kelly S. TransRate: reference-free quality assessment of de novo transcriptome assemblies. Genome Res. 2016:26:1134-44

60. Tatusov RL, Fedorova ND, Jackson JD, Jacobs AR, Kiryutin B, Koonin EV, et al The COG database: an updated version includes eukaryotes. BMC Bioinformatics. 2003:4:41

61. Conesa A, Götz S, García-Gómez JM, Terol J, Talón M, Robles M. Blast2GO: a universal tool for annotation, visualization and analysis in functional genomics research. Bioinformatics. 2005;21:3674-6.

62. Ye J, Fang L, Zheng H, Zhang Y, Chen J, Zhang Z, et al. WEGO: a web tool for plotting GO annotations. Nucleic Acids Res. 2006;34:W293-W7.

63. Moriya Y, Itoh M, Okuda S, Yoshizawa AC, Kanehisa M. KAAS: an automatic genome annotation and pathway reconstruction server. Nucleic Acids Res. 2007:35:W182-W5.

64. Li A, Zhang J, Zhou Z. PLEK: a tool for predicting long non-coding RNAs and messenger RNAs based on an improved k-mer scheme. BMC Bioinformatics. 2014;15:311.

65. Kong $L$, Zhang $Y$, Ye ZQ, Liu XQ, Zhao SQ, Wei L, et al. CPC: assess the protein-coding potential of transcripts using sequence features and support vector machine. Nucleic Acids Res. 2007:35:W345-W9.

66. Wang L, Park HJ, Dasari S, Wang S, Kocher J-P, Li W. CPAT: coding-potential assessment tool using an alignment-free logistic regression model. Nucleic Acids Res. 2013;41:e74

67. Min XJ, Butler G, Storms R, Tsang A. OrfPredictor: predicting protein-coding regions in EST-derived sequences. Nucleic Acids Res. 2005;33:W677-W80. 
68. Gupta V. GitHub repository. https://github.com/vikas0633/perl/blob/master/ orffinder.pl. Accessed Apr 2016

69. Gasteiger E, Gattiker A, Hoogland C, Ivanyi I, Appel RD, Bairoch A. ExPASy: the proteomics server for in-depth protein knowledge and analysis. Nucleic Acids Res. 2003;31:3784-8.

70. Marchler-Bauer A, Derbyshire MK, Gonzales NR, Lu S, Chitsaz F, Geer LY, et al. CDD: NCBI's conserved domain database. Nucleic Acids Res. 2015;43: D222-D6.

71. Finn RD, Coggill P, Eberhardt RY, Eddy SR, Mistry J, Mitchell AL, et al. The Pfam protein families database: towards a more sustainable future. Nucleic Acids Res. 2016;44:D279-D85.

72. Petersen TN, Brunak S, von Heijne G, Nielsen H. SignalP 4.0: discriminating signal peptides from transmembrane regions. Nat Methods. 2011;8:785-6.

73. Käll L, Krogh A, Sonnhammer EL. Advantages of combined transmembrane topology and signal peptide prediction - the Phobius web server. Nucleic Acids Res. 2007:35:W429-W32.

74. Krogh A, Larsson B, Von Heijne G, Sonnhammer ELL. Predicting transmembrane protein topology with a hidden Markov model: application to complete genomes. J Mol Biol. 2001;305:567-80.

75. Li W, Godzik A. Cd-hit: a fast program for clustering and comparing large sets of protein or nucleotide sequences. Bioinformatics. 2006:22:1658-9.

76. Simão FA, Waterhouse RM, loannidis P, Kriventseva EV, Zdobnov EM. BUSCO: assessing genome assembly and annotation completeness with single-copy orthologs. Bioinformatics. 2015;31:3210-2.

77. Wang H, Nuttall PA. Immunoglobulin-G binding proteins in the ixodid ticks, Rhipicephalus appendiculatus, Amblyomma variegatum and Ixodes hexagonus. Parasitology. 1995;111:161-5.

78. Paesen GC, Adams PL, Harlos K, Nuttall PA, Stuart DI. Tick histamine-binding proteins: isolation, cloning, and three-dimensional structure. Mol Cell. 1999; 3:661-71.

79. Bishop R, Lambson B, Wells C, Pandit P, Osaso J, Nkonge C, et al. A cement protein of the tick Rhipicephalus appendiculatus, located in the secretory $e$ cell granules of the type III salivary gland acini, induces strong antibody responses in cattle. Int J Parasitol. 2002;32:833-42.

80. Trimnell AR, Hails RS, Nuttall PA. Dual action ectoparasite vaccine targeting 'exposed' and 'concealed' antigens. Vaccine. 2002;20:3560-8.

81. Mulenga A, Tsuda A, Onuma M, Sugimoto C. Four serine proteinase inhibitors (serpin) from the brown ear tick, Rhiphicephalus appendiculatus; cDNA cloning and preliminary characterization. Insect Biochem Mol Biol. 2003;33:267-76.

82. Paesen GC, Siebold C, Harlos K, Peacey MF, Nuttall PA, Stuart DI. A tick protein with a modified Kunitz fold inhibits human tryptase. J Mol Biol. 2007:368:1172-86

83. Paesen GC, Siebold C, Dallas ML, Peers C, Harlos K, Nuttall PA, et al. An ionchannel modulator from the saliva of the brown ear tick has a highly modified Kunitz/BPTI structure. J Mol Biol. 2009;389:734-47.

84. Preston SG, Majtán J, Kouremenou C, Rysnik O, Burger LF, Cabezas-Cruz A, et al. Novel immunomodulators from hard ticks selectively reprogramme human dendritic cell responses. PLoS Pathog. 2013:9:e1003450.

85. Langmead B, Salzberg SL. Fast gapped-read alignment with bowtie 2. Nat Methods. 2012;9:357-9.

86. Li B, Dewey CN. RSEM: accurate transcript quantification from RNA-Seq data with or without a reference genome. BMC Bioinformatics. 2011;12:323.

87. Robinson MD, Oshlack A. A scaling normalization method for differential expression analysis of RNA-seq data. Genome Biol. 2010;11:R25.

88. Wagner GP, Kin K, Lynch VJ. Measurement of mRNA abundance using RNAseq data: RPKM measure is inconsistent among samples. Theory Biosci. 2012;131:281-5

89. Robinson MD, McCarthy DJ, Smyth GK. edgeR: a bioconductor package for differential expression analysis of digital gene expression data. Bioinformatics. 2010;26:139-40.

90. Schwarz A, Tenzer S, Hackenberg M, Erhart J, Gerhold-Ay A, Mazur J, et al. A systems level analysis reveals transcriptomic and proteomic complexity in Ixodes ricinus midgut and salivary glands during early attachment and feeding. Mol Cell Proteomics. 2014;13:2725-35.

91. Tirloni L, Reck J, Terra RMS, Martins JR, Mulenga A, Sherman NE, et al. Proteomic analysis of cattle tick Rhipicephalus (Boophilus) microplus saliva: a comparison between partially and fully engorged females. PLoS One. 2014;9:e94831.

92. Kim TK, Tirloni L, Pinto AFM, Moresco J, Yates JR III, da Silva Vaz Jr I, et al. Ixodes scapularis tick saliva proteins sequentially secreted every $24 \mathrm{~h}$ during blood feeding. PLoS Negl Trop Dis. 2016;10:e0004323.
93. Aljamali MN, Ramakrishnan VG, Weng H, Tucker JS, Sauer JR, Essenberg RC. Microarray analysis of gene expression changes in feeding female and male lone star ticks, Amblyomma americanum (L). Arch Insect Biochem Physiol. 2009:71:236-53.

94. Bullard RL, Williams J, Karim S. Temporal gene expression analysis and RNA silencing of single and multiple members of gene family in the lone star tick Amblyomma americanum. PLoS One. 2016;11:e0147966.

95. Deitsch KW, Lukehart SA, Stringer JR. Common strategies for antigenic variation by bacterial, fungal and protozoan pathogens. Nature Rev Microbiol. 2009;7:493-503.

96. Xiang FY, Zhou YZ, Zhou JL. Identification of differentially expressed genes in the salivary gand of Rhipicephalus haemaphysaloides by the suppression subtractive hybridization approach. J Integr Agric. 2012;11:1528-36.

97. Wang H, Paesen GC, Nuttall PA, Barbour AG. Male ticks help their mates to feed. Nature. 1998:391:753-4

98. Feldman-Muhsam B, Borut S, Saliternik-Givant S. Salivary secretion of the male tick during copulation. J Insect Physiol. 1970;16:1945-9.

99. Findlay GD, Yi X, MacCoss MJ, Swanson WJ. Proteomics reveals novel Drosophila seminal fluid proteins transferred at mating. PLoS Biol. 2008;6:e178.

100. Sonenshine DE, Bissinger BW, Egekwu N, Donohue KV, Khalil SM, Roe RM. First transcriptome of the testis-vas deferens-male accessory gland and proteome of the spermatophore from Dermacentor variabilis (Acari: Ixodidae). PLoS One. 2011;6:e24711.

101. Adamson SW, Browning RE, Budachetri K, Ribeiro JMC, Karim S. Knockdown of selenocysteine-specific elongation factor in Amblyomma maculatum alters the pathogen burden of Rickettsia parkeri with epigenetic control by the Sin3 histone deacetylase corepressor complex. PLoS One. 2013;8:e82012.

102. Cabezas-Cruz A, Alberdi P, Ayllón N, Valdés JJ, Pierce R, Villar M, et al. Anaplasma phagocytophilum increases the levels of histone modifying enzymes to inhibit cell apoptosis and facilitate pathogen infection in the tick vector Ixodes scapularis. Epigenetics. 2016;11:303-19.

103. Ayllón N, Villar M, Galindo RC, Kocan KM, Šíma R, López JA, et al. Systems biology of tissue-specific response to Anaplasma phagocytophilum reveals differentiated apoptosis in the tick vector Ixodes scapularis. PLoS Genet. 2015:11:e1005120.

104. Kung JTY, Colognori D, Lee JT. Long noncoding RNAs: past, present, and future. Genetics. 2013;193:651-69.

105. Mercer TR, Mattick JS. Structure and function of long noncoding RNAs in epigenetic regulation. Nat Struct Mol Biol. 2013;20:300-7.

106. Engreitz JM, Ollikainen N, Guttman M. Long non-coding RNAs: spatial amplifiers that control nuclear structure and gene expression. Nat Rev Mol Cell Biol. 2016:17:756-70.

107. Dinger ME, Pang KC, Mercer TR, Mattick JS. Differentiating protein-coding and noncoding RNA: challenges and ambiguities. PLoS Comp Biol. 2008;4: e1000176.

108. Aune TM, Spurlock CF. Long non-coding RNAs in innate and adaptive immunity. Virus Res. 2016;212:146-60.

109. Zhang Y, Cao X. Long noncoding RNAs in innate immunity. Cell Mol Immunol. 2016;13:138-47.

110. Etebari K, Asad S, Zhang G, Asgari S. Identification of Aedes aegypti long intergenic non-coding RNAs and their association with Wolbachia and denque virus infection. PLoS Negl Trop Dis. 2016;10:e0005069.

111. Rossetto CC, Pari GS. Kaposi's sarcoma-associated herpesvirus noncoding polyadenylated nuclear RNA interacts with virus-and host cell-encoded proteins and suppresses expression of genes involved in immune modulation. J Virol. 2011:85:13290-7.

112. Scaria V, Pasha A. Long non-coding RNAs in infection biology. Front Genet. 2013;3:308.

113. Shaw MK, Young AS. Differential development and emission of Theileria parva sporozoites from the salivary gland of Rhipicephalus appendiculatus. Parasitology. 1995;111:153-60.

114. Farkaš R. Apocrine secretion: new insights into an old phenomenon. Biochim Biophys Acta. 1850;2015:1740-50.

115. Maruyama SR, Anatriello E, Anderson JM, Ribeiro JMC, Brandão LG, Valenzuela JG, et al. The expression of genes coding for distinct types of glycine-rich proteins varies according to the biology of three metastriate ticks, Rhipicephalus (Boophilus) microplus, Rhipicephalus sanguineus and Amblyomma cajennense. BMC Genomics. 2010;11:363.

116. Binnington KC, Kemp DH. Role of tick salivary glands in feeding and disease transmission. Adv Parasitol. 1980;18:315-39. 
117. Slovák M, Štibrániová I, Hajnická V, Nuttall PA. Antiplatelet-derived growth factor (PDGF) activity in the saliva of ixodid ticks is linked with their long mouthparts. Parasite Immunol. 2014;36:32-42.

118. Moorhouse DE, Tatchell RJ. The feeding processes of the cattle-tick Boophilus microplus (Canestrini): a study in host-parasite relations. Parasitology. 1966;56:623-32.

119. Yi HY, Chowdhury M, Huang YD, Yu XQ. Insect antimicrobial peptides and their applications. Appl Microbiol Biotechnol. 2014;98:5807-22.

120. Rogelj B, Godin KS, Shaw CE, Ule J. The functions of glycine-rich regions in TDP-43, FUS and related RNA-binding proteins. In: Lorkovic ZJ, editor. RNA Binding Proteins. Austin: Landes Bioscience; 2012. p. 1-17.

121. Trimnell AR, Davies GM, Lissina O, Hails RS, Nuttall PA. A cross-reactive tick cement antigen is a candidate broad-spectrum tick vaccine. Vaccine. 2005 23:4329-41.

122. Wang Z, Gerstein M, Snyder M. RNA-Seq: a revolutionary tool for transcriptomics. Nat Rev Genet. 2009;10:57-63.

123. Bolisetty MT, Rajadinakaran G, Graveley BR. Determining exon connectivity in complex mRNAs by nanopore sequencing. Genome Biol. 2015;16:204.

124. Rhoads A, Au KF. PacBio sequencing and its applications. Genomics Proteomics Bioinformatics. 2015;13:278-89.

125. Dai J, Narasimhan S, Zhang L, Liu L, Wang P, Fikrig E. Tick histamine release factor is critical for Ixodes scapularis engorgement and transmission of the Lyme disease agent. PLoS Path. 2010;6:e1001205.

126. Mulenga A, Macaluso KR, Simser JA, Azad AF. The American dog tick, Dermacentor variabilis, encodes a functional histamine release factor homolog. Insect Biochem Mol Biol. 2003;33:911-9.

127. Kemp DH, Bourne A. Boophilus microplus: the effect of histamine on the attachment of cattle-tick larvae - studies in vivo and in vitro. Parasitology. 1980;80:487-96

128. Bommer UA. Cellular function and regulation of the translationally controlled tumour protein TCTP. Open Allergy J. 2012;5:19-32

129. Randolph SE. Tick ecology: processes and patterns behind the epidemiological risk posed by ixodid ticks as vectors. Parasitology. 2004; 129(Suppl):S37-65

130. Berkvens DL, Pegram RG, Brandt JRA. A study of the diapausing behaviour of Rhipicephalus appendiculatus and R. zambeziensis under quasi-natural conditions in Zambia. Med Vet Entomol. 1995;9:307-15.

131. Nuttall PA, Labuda M. Saliva-assisted transmission of tick-borne pathogens. In: Bowman AS, Nuttall PA, editors. Ticks: biology, disease and control. Cambridge: Cambridge University Press; 2008. p. 205-19.

132. Bishop R, Musoke A, Morzaria S, Gardner M, Nene V. Theileria: intracellular protozoan parasites of wild and domestic ruminants transmitted by ixodid ticks. Parasitology. 2004;129(Suppl.):S271-S83.

\section{Submit your next manuscript to BioMed Central and we will help you at every step:}

- We accept pre-submission inquiries

- Our selector tool helps you to find the most relevant journal

- We provide round the clock customer support

- Convenient online submission

- Thorough peer review

- Inclusion in PubMed and all major indexing services

- Maximum visibility for your research

Submit your manuscript at www.biomedcentral.com/submit 